\title{
Nonlinear hydrodynamics from flow of retarded Green's function
}

\author{
Nabamita Banerjee ${ }^{a}$ and Suvankar Dutta ${ }^{b}$ \\ ${ }^{a}$ Nabamita Banerjee, Institute for Theoretical Physics and Spinoza Institute, Utrecht University, \\ Utrecht, The Netherlands \\ ${ }^{b}$ Suvankar Dutta, Dept. of Physics, Swansea University, \\ Singleton Park, Swansea, SA2 8PP, U.K. \\ E-mail: N.Banerjee@uu.nl, pysd@swan.ac.uk
}

ABSTRACT: We study the radial flow of retarded Green's function of energy-momentum tensor and $R$-current of dual gauge theory in presence of generic higher derivative terms in bulk Lagrangian. These are first order non-linear Riccati equations. We solve these flow equations analytically and obtain second order transport coefficients of boundary plasma. This way of computing transport coefficients has an advantage over usual Kubo approach. The non-linear equation turns out to be a linear first order equation when we study the Green's function perturbatively in momentum. We consider several examples including $W e y l^{4}$ term and generic four derivative terms in bulk. We also study the flow equations for $R$-charged black holes and obtain exact expressions for second order transport coefficients for dual plasma in presence of arbitrary chemical potentials. Finally we obtain higher derivative corrections to second order transport coefficients of boundary theory dual to five dimensional gauge supergravity.

KEYWORDS: Gauge-gravity correspondence, Black Holes in String Theory

ARXIV EPRINT: 1005.2367 


\section{Contents}

1 Introduction and discussion 1

2 Flow of retarded Green's function of energy-momentum tensor 5

2.1 A renormalized response function 8

2.2 Second order transport coefficients from flow equation 8

3 Higher derivative correction to flow equation $\quad 9$

4 Examples: string theory corrections to flow equation $\quad 12$

$\begin{array}{lll}4.1 \text { Weyl }^{4} \text { term } & 12\end{array}$

$\begin{array}{lll}4.2 & \text { Four derivative term } & 14\end{array}$

$\begin{array}{lll}\text { 4.2.1 Exact result for Gauss-Bonnet black hole } & 16\end{array}$

$\begin{array}{llr}5 & \text { Flow equation for charged black holes } & \mathbf{1 8}\end{array}$

$\begin{array}{lll}5.1 & \text { R-charged black holes } & 18\end{array}$

5.2 Charged black holes in higher derivative gravity 21

6 Flow of retarded Green's function of boundary $R$ current 24

A Second order transport coefficients from Kubo formula 27

$\begin{array}{ll}\text { B Equivalence of boundary terms } & 29\end{array}$

C Functions appeared in String theory corrected action 32

D Functions appeared in four derivative action $\quad 32$

E Functions appeared in Higher-derivative Charged black-hole action $\quad 33$

\section{Introduction and discussion}

Fluid/gravity correspondence has become an interesting aspect of current theoretical physics research after beginning of $R H I C$ program in 2000. The experimental data implies that the thermalized matter produced at $R H I C$ are in a new state called the quark-gluon plasma (QGP). The evolution of QGP and hadronic matter in this state can be described by hydrodynamics. The temperature of the gas of quarks and gluons produced at RHIC is approximately $170 \mathrm{MeV}$ which is very close to the confinement temperature of QCD. At this high temperature they are not in the weakly coupled regime of QCD. This new phase 
of nuclear matter is known as the the strongly coupled quark-gluon plasma (sQGP). Obviously to study their properties the usual perturbation theory does not work. One needs different approach to deal with these strongly coupled system.

The holographic hydrodynamics (or fluid/gravity correspondence) is an important tool for understanding some properties of strongly coupled CFTs in terms of the dual AdS black holes physics ${ }^{1}[1]-[25]$.

Low frequency (long wavelength) fluctuations of any interacting quantum field theory should be described by hydrodynamics. Low energy behavior of strongly coupled field theory (with gravity dual) is governed by a weakly coupled black hole space-time in one higher dimension. On the other hand in classical theory of gravity the "membrane-paradigm" approach of [26] tells us that a black hole has a fictitious fluid membrane (with hydrodynamic characteristic) living on the horizon of the black hole. Therefore from UV/IR point of view it would be interesting to understand the precise relation between the membrane fluid and the low frequency description of strongly coupled field theory sitting at the boundary of AdS black hole space-time.

One can read off the transport coefficients like shear-viscosity coefficients, of boundary plasma from its retarded Green's function of stress tensor

$$
G_{x y, x y}^{R}\left(k_{\mu}\right)=-i \int d t d x e^{i k \cdot x}\left\langle\left[T_{x y}(x), T_{x y}(0)\right]\right\rangle .
$$

The Green's function for a fluid at finite temperature has a hydrodynamic expansion of the form, ${ }^{2}$

$$
G_{x y, x y}^{R}\left(k_{\mu}\right)=-i \eta \omega+\eta \tau_{\pi} \omega^{2}-\frac{\kappa}{2}\left[(p-2) \omega^{2}+q^{2}\right]+\mathcal{O}\left(k^{3}\right),
$$

where, $p$ is spatial dimension and $p \geq 3$ and

$$
\begin{aligned}
\eta & : \text { shear viscosity coefficient, } \\
\tau_{\pi} & : \text { relaxation time for shear viscous stress, } \\
\kappa & : \text { a new coefficient defined in } \operatorname{Ref}[9,10]
\end{aligned}
$$

and $\omega$ is frequency and $q$ is spatial momentum $(k=\{\omega, 0,0, q\})$.

In [27] the author defined a response function $\bar{\chi}(r, k)$ (eq. (2.13) in our case) it was shown that, at low momentum $\left(k_{\mu} \rightarrow 0\right)$, the evolution of the response function is independent of the radial direction and hence, it can be computed either at horizon or at boundary. Computing the response function at black hole horizon one can show that the shear viscosity coefficient, which is a first-order transport coefficient of the boundary plasma, can be obtained from the low frequency characteristics of the membrane fluid. In [28-30], this issue has been generalized for gravity theory with generic higher-derivative interactions in arbitrary backgrounds. It has been proved that the membrane fluid does give the correct shear-viscosity coefficient of the boundary plasma in arbitrary higher derivative gravity and just the knowledge of near-horizon geometry of the dual AdS black hole is enough for computing the shear-viscosity coefficient [31].

\footnotetext{
${ }^{1} \mathrm{QCD}$ is approximately conformal at sufficiently large energies.

${ }^{2}$ Here we have dropped the frequency independent part of the Green's function.
} 


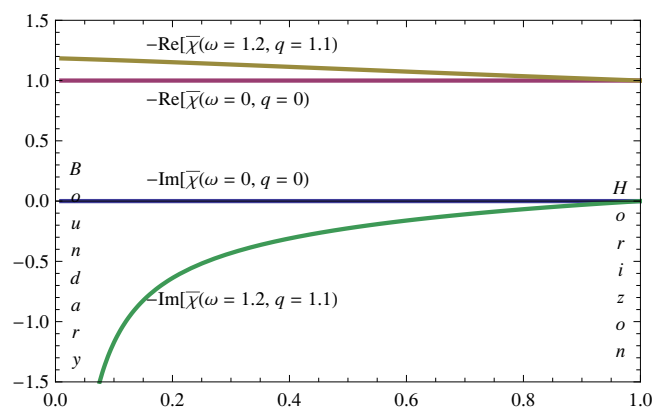

Figure 1. Flow of Green's function from horizon to boundary for two derivative gravity.

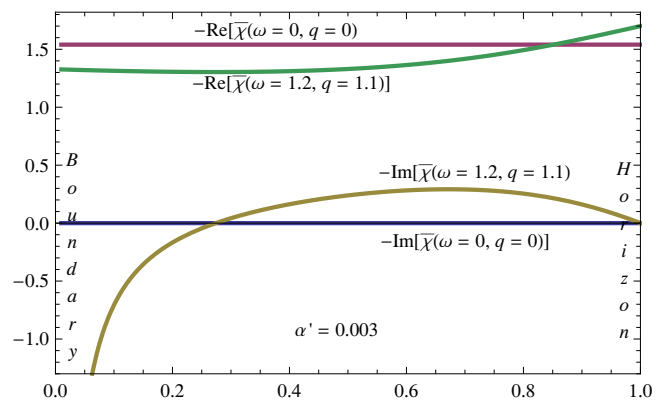

Figure 2. Flow of Green's function from Horizon to boundary for higher derivative gravity for different values of $\omega$ and $q$.

To specify the boundary plasma completely one also needs to understand its higherorder transport coefficients. For this one needs to move away from the low frequency $\left(k_{\mu} \rightarrow 0\right)$ limit. In this case, the response functions flow non-trivially with the radial direction and the flow depends on full black hole geometry. Although the boundary plasma and the membrane fluid have same shear-viscosity coefficients, other transport coefficients can certainly differ and it is not clear how the two are related. In figure 1 we plot the radial evolution of response function for two derivative gravity. ${ }^{3}$ In zero frequency limit, the flow of the response function $\bar{\chi}$ is trivial. Its real part is constant (the constant is one in our scaling) and imaginary part is zero. For finite $\omega$ and $q$ the response function (both real and imaginary part) has a non-trivial evolution. But the horizon value of the function for nonzero frequency is same as the horizon value of the function for zero frequency. Therefore we conclude that for two derivative gravity dual, the full momentum response at the horizon automatically corresponds to only the zero momentum limit of the boundary response.

However in presence of higher derivative terms in the action the situation is different. The full momentum response at horizon depends on spatial momentum. ${ }^{4}$ We plot the flow of response function for $\mathrm{Weyl}^{4}$ interaction in figure 2 .

From this plot we see that in presence of higher-derivative interaction the horizon value of the response function depends of $k_{\mu}$ unlike two derivative theory.

\footnotetext{
${ }^{3}$ We define the response function $\bar{\chi}=\frac{-G_{x y, x y}^{R}(\omega, q)}{i \omega}$.

${ }^{4} \mathrm{We}$ will also see this analytically in sections 3 .
} 
We also see that the imaginary part of $\bar{\chi}$ diverges at boundary. This is the usual $U V$ divergence. One has to add a counter term to cancel this divergence.

In this paper, we study the flow equations for retarded Green's function (in fact response function ${ }^{5}$ ) of boundary theory analytically and find higher order transport coefficients of the boundary plasma solving this equation. We generalize the analysis for generic higher derivative gravity theory and also for $R$ charge black holes. The flow equation for Green's function is a first order non-linear differential equation of Riccati type. Because of its non-linear nature it is hard to solve this equation exactly. After a change of variable one can reduce this non-linear equation to a second order linear homogeneous differential equation. But to solve this we need to specify two boundary conditions. In this paper we deal with the non-linear equation and specify the boundary condition at the horizon. Therefore the hydrodynamic characteristic of the field theory at $U V$ fix point is determined by $I R$ boundary condition.

For two derivative Einstein-Hilbert action the flow equation of retarded Green's function has been derived in [27]. But it is not obvious how to generalize the flow equation for higher derivative gravity. The derivation given in [27] was based on the canonical form of graviton's action. In this paper we have considered generic higher derivatives terms in the bulk Lagrangian. Following the prescription given in [29] we construct an effective action for transverse graviton which has the canonical form in presence of any higher derivative terms in the bulk, and derive the flow equation for Green's function. Solving this flow equation perturbatively in $\omega$ and $q$ we obtain second order transport coefficients namely $\tau_{\pi}$ (relaxation time) and $\kappa$ of the dual plasma. ${ }^{6}$ In this way of computing the transport coefficients has an advantage over usual Kubo approach. In Kubo approach, one has to first find the transverse graviton by solving a second order differential equation and then compute regarded Green's function. Instead, the flow equation is a first order differential equation (although non-linear). As we want a perturbative expansion of Green's function in powers of $\omega$ and $q$ the equation turns out to be a linear first order differential equation. Thus, technically, it is simpler to get results for causal hydrodynamics, particularly when the dual bulk theory is complicated.

The paper is organized as follows. We have worked in five-dimensional bulk theory, the dual gauge theory is four-dimensional. In section 2 we review the derivation of flow equation of boundary Green's function for two derivative gravity. Then solving this flow equation we compute second order transport coefficients $\kappa$ and $\tau_{\pi}$ of boundary plasma. Our results matches with previous computations of $[9,10]$. In section 3 we present the flow equation in presence of generic higher derivative interaction. We calculate the higher derivative correction for $\tau_{\pi}$ and $\kappa$ in section 4 . We concentrated mainly on $W e y l^{4}$ and four derivative correction. While our results for $\mathrm{Weyl}^{4}$ correction are in agreement with results

\footnotetext{
${ }^{5}$ The Green's function, by definition, is independent of $r$. Therefore whenever we say flow of Green's function we mean the flow of the quantity defined in equation (2.12) before taking $r \rightarrow 0$ limit (which is defined to be response function up to a factor of $i \omega$ ).

${ }^{6}$ From Weyl invariance one can show that there are other transport coefficients in second order hydrodynamics [10]. However from the expansion of retarded Green's function it is only possible to compute only two of them.
} 
of [32], the four-derivative corrections are new results of this paper. We also compute the effect of gauss-Bonnet to $\tau_{\pi}$ and $\kappa$. From our results we obtain the bound on Gauss-Bonnet coupling constant. In section 5 we consider $R$ charge black hole in bulk and find the second order transport coefficients in presence of finite chemical potential. We also find the higher derivative effects on transport coefficients of field theory plasma dual to gauge supergravity theory. Finally,we analyze the flow equation of Green's function for boundary $R$ current in section 6. Appendix A and B compliments some discussions on boundary terms. In appendix $\mathrm{C}, \mathrm{D}$ and $\mathrm{E}$, we have provided some long expressions.

\section{Flow of retarded Green's function of energy-momentum tensor}

In this section we briefly review the work of Liu and Iqbal [27]. They considered leading Einstein-Hilbert (E-H) action with a negative cosmological constant in $4+1$ dimensions and studied the motion of a transverse graviton in this background. ${ }^{7}$ The action is,

$$
S_{\mathrm{EM}}=\frac{1}{16 \pi G_{5}} \int d^{5} x \sqrt{-g}(R+12) .
$$

The background has a black-brane solution as,

$$
\begin{aligned}
d S^{2} & =g_{t t} d t^{2}+g_{r r} d r^{2}+g_{i j} d x^{2} d x^{j} \\
g_{t t} & =-\frac{1-r^{2}}{r}, \quad g_{r r}=\frac{1}{4 r^{2}\left(1-r^{2}\right)} \\
g_{i j} & =\frac{1}{r} \delta_{i j} .
\end{aligned}
$$

The solution is asymptotically AdS and it has a boundary topology $R \times R^{3}$. The horizon of the space-time is at $r \rightarrow 1$ and asymptotic boundary is at $r \rightarrow 0$.

We study the graviton's fluctuation in this background,

$$
g_{x y}=g_{x y}^{(0)}+h_{x y}(r, x)=g_{x y}^{(0)}[1+\epsilon \Phi(r, x)] .
$$

By plugging it in the action and keeping terms to order $\epsilon^{2}$, we obtain the following effective action for the perturbation

$$
S=\frac{1}{16 \pi G_{5}} \int \frac{d^{4} k}{(2 \pi)^{4}} d r \sum_{p, q=0}^{2} \mathcal{A}_{p, q}(r, k) \phi^{(p)}(r,-k) \phi^{(q)}(r, k) .
$$

Here we use Fourier transform to work in the momentum space $k=\{-\omega, \vec{k}\}$,

$$
\Phi(r, x)=\int \frac{d^{4} k}{(2 \pi)^{4}} e^{i k \cdot x} \phi(r, k)
$$

and $\phi^{(p)}(r, k)$ denotes the $p^{t h}$ derivative of the field $\phi(r, k)$ with respect to $r(p+q \leq 2)$.

\footnotetext{
${ }^{7}$ Here we restrict ourselves to five space-time dimensions, but the discussions are quite generic and can be extended to arbitrary dimensions.
} 
Next, we integrate by parts to obtain the bulk action in the following form (up to some total derivative terms)

$$
S=\int \frac{d^{4} k}{(2 \pi)^{4}} d r\left(\mathcal{A}_{1}(r, k) \phi^{\prime}(r, k) \phi^{\prime}(r,-k)+\mathcal{A}_{0}(r, k) \phi(r, k) \phi(r,-k)\right)
$$

where,

$$
\begin{aligned}
& \mathcal{A}_{1}(r, k)=-\frac{\frac{1}{2} g^{r r} \sqrt{-g}}{16 \pi G_{5}}, \\
& \mathcal{A}_{0}(r, k)=-\frac{\frac{1}{2} \sqrt{-g} g^{\mu \nu} k_{\mu} k_{\nu}}{16 \pi G_{5}} .
\end{aligned}
$$

From this action, we can find the conjugate momentum $\Pi\left(r, k_{\mu}\right)$ of the transverse graviton (for r-foliation) and the equation of motion,

$$
\Pi\left(r, k_{\mu}\right)=2 \mathcal{A}_{1}(r, k) \phi^{\prime}(r, k)
$$

and

$$
\Pi^{\prime}\left(r, k_{\mu}\right)-2 \mathcal{A}_{0}(r, k) \phi(r, k)=0 .
$$

The on-shell action reduces to the following surface term, ${ }^{8}$

$$
S=\sum_{r=0,1} \int \frac{d^{4} k}{(2 \pi)^{4}}\left(\mathcal{A}_{1}(r, k) \phi^{\prime}(r, k) \phi(r,-k)\right) .
$$

Following the AdS/CFT prescription given in [5], the boundary retarded Green's function is given as,

$$
G_{R}\left(k_{\mu}\right)=\lim _{r \rightarrow 0} \frac{2 \mathcal{A}_{1}(r, k) \phi^{\prime}(r, k) \phi(r,-k)}{\phi_{0}(k) \phi_{0}(-k)},
$$

where, $\phi_{0}\left(k_{\mu}\right)$ is the value of the graviton fluctuation at boundary. Full solution of the graviton can be written as $\phi\left(r, k_{\mu}\right)=\phi_{0}\left(k_{\mu}\right) F\left(r, k_{\mu}\right)$, where $F\left(r, k_{\mu}\right)$ goes to identity at the boundary. We can rewrite the boundary retarded Green's function as,

$$
G_{R}\left(k_{\mu}\right)=\lim _{r \rightarrow 0} \frac{\Pi\left(r, k_{\mu}\right)}{\phi\left(r, k_{\mu}\right)} .
$$

Let us define a response function of the boundary theory as, ${ }^{9}$

$$
\bar{\chi}\left(k_{\mu}, r\right)=\frac{\Pi\left(r, k_{\mu}\right)}{i \omega \phi\left(r, k_{\mu}\right)}
$$

where $\omega=k_{0}$. This function is defined for all $r$ and $k_{\mu}$. Therefor the boundary Green's function is given by,

$$
G_{R}\left(k_{\mu}\right)=\lim _{r \rightarrow 0} i \omega \bar{\chi}\left(k_{\mu}, r\right) .
$$

\footnotetext{
${ }^{8}$ We will discuss about other boundary terms in appendix B.

${ }^{9}$ We set the zero frequency part of $\mathrm{G}$ to zero, as it gives contact terms.
} 
We will study the radial evolution of the response function $\bar{\chi}\left(k_{\mu}\right)$ from horizon to boundary. Differentiating equation (2.13) and using the equations of motion (2.9) we get,

$$
\partial_{r} \bar{\chi}\left(k_{\mu}, r\right)=i \omega \sqrt{-\frac{g_{r r}}{g_{t t}}}\left[\frac{\bar{\chi}\left(k_{\mu}, r\right)^{2}}{\Sigma(r)}-\frac{\Upsilon(r)}{\omega^{2}}\right],
$$

where we define

$$
\begin{aligned}
& \Sigma(r)=-2 \mathcal{A}_{1}\left(r, k_{\mu}\right) \sqrt{-\frac{g_{r r}}{g_{t t}}} \\
& \Upsilon(r)=2 \mathcal{A}_{0}\left(r, k_{\mu}\right) \sqrt{-\frac{g_{t t}}{g_{r r}}} .
\end{aligned}
$$

Putting values of $\mathcal{A}_{1}$ and $\mathcal{A}_{0}$ given in (2.7) we can easily recover the flow equation given in [27]. ${ }^{10}$ However for future requirements, here we present it directly in terms of the coefficients of the graviton action.

As mentioned earlier, the flow equation in (2.15) is valid for any value of momentum. This is a first order differential equation and we need to specify one boundary condition to solve this equation. That naturally comes from the behavior of the equation at the horizon. Demanding the solution to be regular at the horizon, we get the following condition,

$$
\left.\bar{\chi}\left(k_{\mu}, r\right)^{2}\right|_{r=1}=\left.\frac{\Sigma(r) \Upsilon(r)}{\omega^{2}}\right|_{r=1} .
$$

For two derivative gravity this boundary condition implies that ${ }^{11}$

$$
\bar{\chi}\left(k_{\mu}, 1\right)=-\sqrt{\frac{\Sigma(1) \Upsilon(1)}{\omega^{2}}}=-\frac{1}{16 \pi G_{5}}
$$

which is independent of $k_{\mu}$. Therefore the full momentum response at the horizon corresponds to only to the zero momentum limit of boundary response, $\bar{\chi}\left(k_{\mu}, 1\right)=\bar{\chi}\left(k_{\mu} \rightarrow\right.$ $0, r \rightarrow 0) .{ }^{12}$

With this boundary condition, one can integrate out the differential equation (2.15) from horizon to asymptotic boundary and obtain the AdS/CFT response for all momentum $k_{\mu}$. In particular, it is trivial to see that at $\left(\omega, k_{i}\right) \rightarrow 0$ limit, the flow is trivial

$$
\partial_{r} \bar{\chi}\left(k_{\mu}, r\right)=0
$$

and using the boundary condition (2.18) we get the first order transport coefficient of boundary fluid, i.e. the shear viscosity coefficient coefficients $\eta=\frac{1}{16 \pi G_{5}}$.

In this paper, we will go away from $\left(\omega, k_{i} \rightarrow 0\right)$ limit. As we have already mentioned, it is possible to integrate the flow equation for any momentum (perturbatively) and we can easily find the higher order transport coefficients. The usual Kubo approach to compute these coefficients requires the full profile of the transverse graviton in black hole background (solving a second order differential equation), where as, using the flow equation, one can get these transport coefficients without explicit knowledge of the graviton's profile.

\footnotetext{
${ }^{10}$ just notice that $g_{t t}$ there is negative of what we used here.

${ }^{11}$ We choose the negative brunch. The sign of the boundary condition 2.18 depends on the choice of coordinate. In our coordinate the boundary is at $r \rightarrow 0$ hence we need to choose the negative branch.

${ }^{12}$ However, in higher derivative gravity we will see that the $\bar{\chi}\left(k_{\mu}, 1\right)$ depends on spatial momentum.
} 


\subsection{A renormalized response function}

When we solve the flow equation (2.15) to get the boundary response function in general it involves divergence at the boundary $(r \rightarrow 0)$. These are usual $U V$ divergences and to remove them we need to re-normalize the response function properly.

We follow the holographic renormalization prescription of $[34,35]$. As the graviton is massless, we only need to add the following counterterm to the graviton's action,

$$
S_{C}=\frac{1}{16 \pi G_{5}} \int_{r=\delta} d^{4} x \sqrt{-\gamma} \frac{1}{4} \Phi(\epsilon, x) \square \Phi(\epsilon, x) .
$$

In momentum space,

$$
S_{C}=\frac{1}{64 \pi G_{5}} \int_{r=\delta} \frac{d^{4} k}{(2 \pi)^{4}} \sqrt{-\gamma} \phi(\delta, k)\left(g^{t t} \omega^{2}+k_{i} k^{i}\right) \phi(\delta,-k) .
$$

Therefore the renormalized Green's function is given by,

$$
\mathcal{G}_{R}=\lim _{r \rightarrow 0}\left[\frac{\Pi\left(r, k_{\mu}\right)}{\phi\left(r, k_{\mu}\right)}+\frac{\sqrt{-\gamma}}{32 \pi G_{5}}\left(g^{t t} \omega^{2}+k_{i} k^{i}\right)\right] .
$$

However we will study the flow of un-renormalized response function defined in (2.13) and we define our renormalized response function as,

$$
\bar{\chi}^{\operatorname{Ren}}\left(r, k_{\mu}\right)=\bar{\chi}\left(r, k_{\mu}\right)+\frac{1}{i \omega} \frac{\sqrt{-\gamma}\left(g^{t t} \omega^{2}+k_{i} k^{i}\right)}{32 \pi G_{5}} .
$$

The counter term will cancel the UV divergences appearing in the expression of $\bar{\chi}$ and we will get a finite result at the boundary, i.e. $\lim _{r \rightarrow 0} \bar{\chi}^{\operatorname{Ren}}\left(r, k_{\mu}\right)$ will be finite. From the above analysis, we understand that one can get rid of the UV divergences appearing in the response function by following the holographic renormalization technique. But, an important observation is, this counter term does not add any finite contribution to the result it only cancels out the divergences. Thus, one can study the flow of the un-renormalized response function and ignore the divergences piece to get the finite contribution at the boundary.

\subsection{Second order transport coefficients from flow equation}

In this subsection, we compute the higher order transport coefficients by solving the flow equation (2.15) perturbatively up to order $\omega^{2}$ and $k_{i}^{2}$. This is a non-linear first order differential equation. Now, the right hand side of this equation is proportional to $\omega$. Hence, to solve $\bar{\chi}$ to order $\omega^{2}$, we can replace the leading order solution for $\bar{\chi}$ in the right hand side of equation (2.15). This simplifies the situation a lot as the non-linear equation becomes linear. Now, to leading order, $\bar{\chi}=-\eta=-\frac{1}{16 \pi G_{5}}$. Therefore up to order $\omega^{2}$, we get,

$$
\partial_{r} \bar{\chi}\left(k_{\mu}, r\right)=i \omega \sqrt{-\frac{g_{r r}}{g_{t t}}}\left[\frac{\eta^{2}}{\Sigma(r)}-\frac{\Upsilon(r)}{\omega^{2}}\right]+\mathcal{O}\left(\omega^{2}, k_{i}^{2}\right) .
$$


The integration constant for the equation can be fixed form the boundary condition (2.18). Putting the value of the constant, the solution takes the form,

$$
\begin{aligned}
i \omega \bar{\chi}\left(k_{\mu}, 0\right)= & \lim _{r \rightarrow 0}-\frac{1}{96 \pi G_{5} r}\left[3 q^{2}(r-1)+\omega(3 \omega+r(\omega(\log (8)-3)+6 i))\right] \\
& +\mathcal{O}\left(q \omega^{2}, \omega q^{2}, q^{3}, \omega^{3}\right) \\
= & -i \omega\left(\frac{1}{16 \pi G_{5}}\right)+\omega^{2}\left[\frac{1}{2}(1-\ln 2)\left(\frac{1}{16 \pi G_{5}}\right)\right]-\frac{q^{2}}{2}\left(\frac{1}{16 \pi G_{5}}\right)+\mathcal{O}\left(\frac{1}{r}\right) .
\end{aligned}
$$

Here we have chosen the four momentum in the following form

$$
k=\{\omega, 0,0, q\} .
$$

This expression has divergence as $r \rightarrow 0$ (UV divergence) and can be removed by adding suitable counter term (as explained in the last section).

Comparing the finite piece of (2.26) at $r \rightarrow 0$ with the generic expansion of the retarded Green's function (1.2), ${ }^{13}$ we get,

$$
\begin{aligned}
\eta & =\frac{T^{3} \pi^{3}}{16 \pi G_{5}}, \\
\tau_{\pi} & =\frac{2-\ln 2}{2 \pi T}, \quad \kappa=\frac{\eta}{\pi T} .
\end{aligned}
$$

Here $T=\frac{1}{\pi}$. These results are in agreement with [10]. In appendix A we briefly outline the Kubo method to get this result. Thus, we see that, studying the flow equation of the response function we can compute the higher order transport coefficients perturbatively. Here, we present the results for the second order transport coefficients, but, in general it is possible to go beyond second order.

At this point, it is not clear why only considering the boundary term from action (2.6) is enough to get the correct results. In usual Kubo approach, one needs to take into account the Gibbons-Hawking term also. But as the action (2.6) has well defined variational principle, one does not need to add any Gibbons-Hawking term with it. In appendix B we show that the boundary terms coming from the original action and the corresponding Gibbons-Hawking action are exactly same as the boundary terms coming from the action (2.6) up to terms proportional to $\phi^{2}$ and pure divergence terms. The $\phi^{2}$ terms do not contribute to any transport coefficients. ${ }^{14}$ The divergent terms will get canceled by the proper counterterms and hence are not important for finding the transport coefficients. Thus it is clear that the effective action will give us the correct transport coefficients for the boundary plasma. This observation also holds for higher derivative gravity theory. ${ }^{15}$

\section{Higher derivative correction to flow equation}

So far we have discussed the flow equation of two point correlation function of energymomentum tensor of boundary theory whose gravity dual is given by Einstein-Hilbert

\footnotetext{
${ }^{13}$ The overall sign depends on the choice of coordinate.

${ }^{14}$ They only contribute to pressure of the boundary theory.

${ }^{15}$ In appendix B, we have proved this explicitly for $R^{(n)}$ (contraction of $n$ curvature tensors) gravity theory.
} 
action (two derivative action). But it is not obvious how to generalize this for higher derivative case. The proof given in [27] was based on the canonical form (2.6) of graviton's action. In presence of arbitrary higher derivative terms in the bulk, the general action for the perturbation $h_{x y}$ does not have the above form (2.6). Rather it will have more than two derivative (with respect to $r$ ) terms like $\phi^{\prime} \phi^{\prime \prime}, \phi^{\prime \prime 2}$ etc. In presence of these terms it is not possible to bring this action into a canonical form (up to some total derivative terms). In this paper we consider generic higher derivatives terms in the bulk Lagrangian. We follow the prescription of [29] to construct an effective action " $S_{\text {eff }}$ " for transverse graviton in canonical form in presence of generic higher derivative terms in the bulk. The effective action and original action give same equation of motion perturbatively in the coupling of the higher derivative terms.

Let us consider a gravity set-up with $n$ derivative action.

$$
\mathcal{I}=\frac{1}{16 \pi G_{5}} \int d^{5} x \sqrt{-g}\left[R+12+\alpha^{\prime} R^{(n)}\right]
$$

where, $\mathcal{R}^{(n)}$ is any $n$ derivative Lagrangian. The metric in general is given by (assuming planar symmetry),

$$
\begin{aligned}
d s^{2}= & -\left(h_{t}(r)+\alpha^{\prime} h_{t}^{(n)}(r)\right) d t^{2}+\frac{d r^{2}}{h_{r}(r)+\alpha^{\prime} h_{r}^{(n)}(r)} \\
& +\frac{1}{r}\left(1+\alpha^{\prime} h_{s}^{(n)}(r)\right) d \vec{x}^{2} .
\end{aligned}
$$

Substituting the background metric with fluctuation (2.3) in action (3.1) (we call it general action or original action) for the scalar field $\phi(r, k)$ we get,

$$
\mathcal{I}=\frac{1}{16 \pi G_{5}} \int \frac{d^{4} k}{(2 \pi)^{4}} d r \sum_{p, q=0}^{n} \mathcal{A}_{p, q}(r, k) \phi^{(p)}(r,-k) \phi^{(q)}(r, k)
$$

where, $\phi^{(p)}(r, k)$ denotes the $p^{\text {th }}$ derivative of the field $\phi(r, k)$ with respect to $r$ and $p+q \leq$ $n$. The coefficients $\mathcal{A}_{p, q}(r, k)$ in general depends on the coupling constant $\alpha^{\prime}$. $\mathcal{A}_{p, q}$ with $p+q \geq 3$ are proportional to $\alpha^{\prime}$ and vanishes in $\alpha^{\prime} \rightarrow 0$ limit, since the terms $\phi^{(p)} \phi^{(q)}$ with $p+q \geq 3$ appears as an effect of higher derivative terms in the action (3.1).

Up to some total derivative terms, the general action (2.6) can also be written as,

$$
\begin{aligned}
& \mathcal{I}=\frac{1}{16 \pi G_{5}} \int \frac{d^{4} k}{(2 \pi)^{4}} d r \sum_{p=0}^{n / 2} \mathcal{A}_{p}(r, k) \phi^{(p)}(r,-k) \phi^{(p)}(r, k) \\
&=\frac{1}{16 \pi G_{5}} \int \frac{d^{4} k}{(2 \pi)^{4}} d r \sum_{p=0}^{\frac{n-1}{2}} \mathcal{A}_{p}(r, k) \phi^{(p)}(r,-k) \phi^{(p)}(r, k) \\
&(\text { for n } \quad \text { odd }) .
\end{aligned}
$$

However this action does not have canonical form. We write an effective action for transverse graviton in canonical form,

$$
S_{\mathrm{eff}}=\frac{1}{16 \pi G_{5}} \int \frac{d^{4} k}{(2 \pi)^{4}} d r\left[\mathcal{A}_{1}^{\mathrm{HD}}(r, k) \phi^{\prime}(r, k) \phi^{\prime}(r,-k)+\mathcal{A}_{0}^{\mathrm{HD}}(r, k) \phi(r, k) \phi(r,-k)\right](3.5
$$


with some unknown functions $\mathcal{A}_{1}^{H D}$ and $\mathcal{A}_{0}^{H D}$. We fix these functions by demanding that the equations of motion obtained from the effective action and the original action are same perturbatively in $\alpha^{\prime}$.

The generalized canonical momentum and equation of motion are given by,

$$
\begin{aligned}
\Pi^{\mathrm{HD}}(r, k) & =2 \mathcal{A}_{1}^{\mathrm{HD}}(r, k) \phi^{\prime}(r, k) \\
\left(\Pi^{\mathrm{HD}}(r, k)\right)^{\prime} & =2 \mathcal{A}_{0}^{\mathrm{HD}}(r, k) \phi(r, k) .
\end{aligned}
$$

Once we find the effective action for the graviton, we follow the procedure in the previous section to obtain the flow equation for the boundary Green's function in generic higher derivative gravity.

The boundary Green's function is given by,

$$
G_{R}^{\mathrm{HD}}\left(k_{\mu}\right)=\lim _{r \rightarrow 0} \frac{2 \mathcal{A}_{1}^{\mathrm{HD}}(r, k) \phi^{\prime}(r, k) \phi(r,-k)}{\phi_{0}(k) \phi_{0}(-k)},
$$

which can be written using the definition of canonical momentum as,

$$
G_{R}^{\mathrm{HD}}\left(k_{\mu}\right)=\lim _{r \rightarrow 0} \frac{\Pi^{\mathrm{HD}}\left(r, k_{\mu}\right)}{\phi\left(r, k_{\mu}\right)} .
$$

Let us define a response function of the boundary theory in higher derivative theory as,

$$
\bar{\chi}^{\mathrm{HD}}\left(k_{\mu}, r\right)=\frac{\Pi^{\mathrm{HD}}\left(r, k_{\mu}\right)}{i \omega \phi\left(r, k_{\mu}\right)} .
$$

Therefore the flow equation is given by,

$$
\partial_{r} \bar{\chi}^{\mathrm{HD}}\left(k_{\mu}, r\right)=i \omega \sqrt{-\frac{g_{r r}}{g_{t t}}}\left[\frac{\bar{\chi}^{\mathrm{HD}}\left(k_{\mu}, r\right)^{2}}{\Sigma^{\mathrm{HD}}(r, k)}-\frac{\Upsilon^{\mathrm{HD}}(r, k)}{\omega^{2}}\right],
$$

where we define

$$
\begin{aligned}
& \Sigma^{\mathrm{HD}}(r, k)=-2 \mathcal{A}_{1}^{\mathrm{HD}}\left(r, k_{\mu}\right) \sqrt{-\frac{g_{r r}}{g_{t t}}} \\
& \Upsilon^{\mathrm{HD}}(r, k)=2 \mathcal{A}_{0}^{\mathrm{HD}}\left(r, k_{\mu}\right) \sqrt{-\frac{g_{t t}}{g_{r r}}} .
\end{aligned}
$$

This is the flow equation for two point correlation function of energy-momentum tensor in presence of generic higher derivative term in the bulk action. Therefore integrating this equation from horizon to asymptotic boundary one can find the higher derivative correction to the transport coefficients at any order in frequency/momentum.

Like two derivative case here also we need to provide a boundary condition to solve this equation. The response function $\bar{\chi}^{\mathrm{HD}}\left(k_{\mu}, r\right)$ should be well-defined at horizon. This implies,

$$
\left.\bar{\chi}^{\mathrm{HD}}\left(k_{\mu}, r\right)\right|_{r=r_{h}}=\left.\sqrt{\frac{\Sigma^{\mathrm{HD}}(r) \Upsilon^{\mathrm{HD}}(r)}{\omega^{2}}}\right|_{r=r_{h}}
$$

here the horizon is located at $r=r_{h}$. 
One important point to mention here is that unlike two derivative gravity where $\bar{\chi}\left(k_{\mu}, r_{h}\right)$ was independent of $k_{\mu}, \bar{\chi}^{\mathrm{HD}}\left(k_{\mu}, r_{h}\right)$ can in general depend on $k_{\mu}$. We will see this explicitly in the next section. Therefore the full momentum response at the horizon may not be able to correspond only to the zero momentum limit of boundary response in higher derivative theory.

Like two derivative case, the response function in higher-derivative gravity theory also contains UV divergences. We need to add proper counter term following the holographic renormalization procedure to cancel these divergences. A little more thinking also says that in presence of any higher-derivative term in the action the structure of the counterterm remains same as (2.21). Only the overall normalization constant depends on higher-derivative coupling. Thus, similar to the leading gravity, the counterterm in higher derivative gravity also cancels out the divergence and does not add any finite contribution to the boundary response function. One can study the flow equation of the un-renormalized response function and read off the transport coefficients from its finite piece.

\section{Examples: string theory corrections to flow equation}

String theory predicts next to leading order corrections to Einstein-Hilbert action. These corrections are relevant at a distance comparable with typical length scale of the theory $l_{s}=\sqrt{\alpha^{\prime}}$. The short distance corrections to this action is described by supplementing this action by higher curvature terms. However, here we treat the stringy effects perturbatively i.e the coupling of higher derivative terms to be small.

From the point of view of AdS/CFT the small $\alpha^{\prime}$ correction in supergravity corresponds to $\frac{1}{\lambda}$ correction in strongly coupled gauge theory in planar limit where $\lambda$ is the 't Hooft coupling. The precise dictionary between string length and 't Hooft coupling is,

$$
\alpha^{\prime 2}=\frac{L^{4}}{4 \pi \lambda}, \quad L \quad A d S \text { radius }
$$

In this section, we will consider two examples of higher-derivative terms coming from string theory and study their effects on flow equations and second order transport coefficients.

\subsection{Weyl ${ }^{4}$ term}

We consider the well known $W e y l^{4}$ term. This term appears in type II string theory. Adding this term in the bulk action corresponds to $\frac{1}{\lambda^{3 / 2}}$ correction in dual large $N$ theory. The string theory correction to second order transport coefficients have already been computed in [32] using usual Kubo formula. Here, we show that one can obtain the correct result by studying the first order flow equation in higher derivative gravity without solving any second order differential equation for graviton.

The five dimensional bulk action is given by,

$$
S=\frac{1}{16 \pi G_{5}} \int d^{5} x \sqrt{-g}\left(R+12+\gamma W^{(4)}\right)
$$


where, the coupling constant $\gamma$ is given by,

$$
\gamma=\frac{1}{8} \zeta(3) \alpha^{\prime 3}
$$

and

$$
W^{(4)}=C^{h m n k} C_{p m n q} C_{h}^{r s p} C_{r s k}^{q}+\frac{1}{2} C^{h k m n} C_{p q m n} C_{h}^{r s p} C_{r s k}^{q}
$$

and the Weyl tensors $C_{a b c d}$ are given by,

$$
C_{a b c d}=R_{a b c d}+\frac{1}{3}\left(g_{a d} R_{c b}+g_{b c} R_{a d}-g_{a c} R_{d b}-g_{b d} R_{c a}\right)+\frac{1}{12}\left(g_{a c} g_{b d}-g_{a d} g_{c b}\right) R .
$$

The background metric is given by $[36,37],{ }^{16}$

$$
\begin{aligned}
d s^{2}= & -\left(\frac{\left(1-r^{2}\right) r_{0}^{2}}{r}-15 r\left(3 r^{6}-8 r^{4}+5\right) \gamma r_{0}^{2}\right) d t^{2} \\
& +\left(\frac{1}{4 r^{2}-4 r^{4}}+\frac{\left(-285 r^{4}+75 r^{2}+75\right) \gamma}{4-4 r^{2}}\right) d r^{2}+\frac{1}{r} d \vec{x}^{2}
\end{aligned}
$$

The temperature of this black hole is given by,

$$
T=\frac{r_{0}}{\pi}(1+15 \gamma)
$$

and the horizon is located at $r=1$.

The effective action for transverse graviton has been computed in [29]. Here we will only present the result. The effective action is given by,

$$
S_{\text {eff }}=\frac{1}{16 \pi G_{5}} \int \frac{d^{4} k}{(2 \pi)^{4}} d r\left[\mathcal{A}_{1}^{W^{4}}(r, k) \phi^{\prime}(r, k) \phi^{\prime}(r,-k)+\mathcal{A}_{0}^{W^{4}}(r, k) \phi(r, k) \phi(r,-k)\right]
$$

where, $\mathcal{A}_{1}^{W^{4}}$ and $\mathcal{A}_{1}^{W^{4}}$ are given in appendix C.

Therefore the flow equation is given by,

$$
\partial_{r} \bar{\chi}^{W^{4}}\left(k_{\mu}, r\right)=i \omega \sqrt{-\frac{g_{r r}}{g_{t t}}}\left[\frac{\bar{\chi}^{W^{4}}\left(k_{\mu}, r\right)^{2}}{\Sigma^{W^{4}}(r, k)}-\frac{\Upsilon^{W^{4}}(r, k)}{\omega^{2}}\right],
$$

where,

$$
\begin{aligned}
& \Sigma^{W^{4}}(r, k)=-2 \mathcal{A}_{1}^{W^{4}}\left(r, k_{\mu}\right) \sqrt{-\frac{g_{r r}}{g_{t t}}} \\
& \Upsilon^{W^{4}}(r, k)=2 \mathcal{A}_{0}^{W^{4}}\left(r, k_{\mu}\right) \sqrt{-\frac{g_{t t}}{g_{r r}}}
\end{aligned}
$$

The explicit expressions for $\Sigma$ and $\Upsilon$ can be obtained by using $\mathcal{A}_{1}^{W^{4}}$ and $\mathcal{A}_{1}^{W^{4}}$.

\footnotetext{
${ }^{16}$ In this particular example we keep the extremality parameter $r_{0}$ explicitly. It would help us to write the relation between different transport coefficients. One can set $r_{0}$ to be one by time re-scaling what we have done in the next example.
} 
From the regularity of $\bar{\chi}^{W^{4}}\left(k_{\mu}, r\right)$ at horizon we get,

$$
\begin{aligned}
\bar{\chi}^{W^{4}}\left(k_{\mu}, 1\right) & =\sqrt{\frac{\Sigma^{W^{4}}(1) \Upsilon^{W^{4}}(1)}{\omega^{2}}} \\
& =\frac{r_{0}^{3}}{16 \pi G_{5}}+\frac{\gamma r_{0}}{4 \pi G_{5}}\left(45 r_{0}^{2}+11 q^{2}\right) .
\end{aligned}
$$

Here, we see that unlike the two-derivative gravity, the horizon value of the response function depends on spatial momenta $q$ (see figure 2). With this boundary condition we solve the flow equation up to order $\omega^{2}$ and $q^{2}$ (ignoring $\mathcal{O}\left(\omega q^{2}\right)$ term). Here we write the final result. ${ }^{17}$

$$
\begin{aligned}
i \omega \bar{\chi}^{W^{4}}\left(k_{\mu}, 0\right)= & -i(1+180 \gamma) \frac{r_{0}^{3}}{16 \pi G_{5}} \omega \\
& +\left[\frac{1}{2}(1-\log (2))+\frac{5}{4} \gamma(199-66 \log (2))\right] \frac{r_{0}^{2}}{16 \pi G_{5}} \omega^{2} \\
& -\frac{1}{2}(1+20 \gamma) \frac{r_{0}^{2}}{16 \pi G_{5}} q^{2}+\mathcal{O}\left(q \omega^{2}, \omega q^{2}, q^{3}, \omega^{3}\right) .
\end{aligned}
$$

Comparing this result with (1.2) we get

$$
\begin{aligned}
\frac{\eta}{\pi^{3} T^{3}} & =1+135 \gamma+\mathcal{O}\left(\gamma^{2}\right) \\
\kappa & =\frac{\eta}{\pi T}(1-145 \gamma)+\mathcal{O}\left(\gamma^{2}\right) \\
\tau_{\pi} T & =\frac{2-\log (2)}{2 \pi}+\frac{375 \gamma}{4 \pi}+\mathcal{O}\left(\gamma^{2}\right) .
\end{aligned}
$$

These results are in agreement with [32]. It provides a non-trivial check to this approach of obtaining higher order transport coefficients from the flow equation (2.15).

\subsection{Four derivative term}

In this section, we will concentrate on the generic four derivative corrections to EinsteinHilbert action. These terms arise in the effective action for the heterotic string theory. In fact, the complete super-symmetrized $R^{2}$ correction to effective Heterotic string theory is known and one way to obtain it is the super-symmetrization of the Lorentz Chern-Simons terms [38, 39]. This terms also arises in the context of Type IIB string theory [40, 41], where the theory is on $A d S_{5} \times X^{5}$, the compact space $X^{5}$ being $S^{5} / Z_{2}$. The dual theory is $\mathcal{N}=2 \mathrm{Sp}(N)$ gauge theory with 4 fundamental and 1 antisymmetric traceless hypermultiplets. This super-conformal theories arises in the context of ND3-branes sitting inside 8 D7-branes coincident on an orientifold 7-plane. In this case, generic four derivative $R^{2}$ correction comes form the DBI action of the branes.

Here we compute the generic four derivative correction to the second order transport coefficients, the relaxation time $\tau_{\pi}$ and $\kappa$. We can choose the coefficients of the higher

\footnotetext{
${ }^{17} k=\{\omega, 0,0, q\}$ and we ignore the UV divergence piece.
} 
derivative terms to be the four-dimensional Euler density and get pure Gauss-Bonnet correction to these coefficients.

The action is

$$
\mathcal{I}=\frac{1}{16 \pi G_{5}} \int d^{5} x \sqrt{-g}\left[R+12+\alpha^{\prime}\left(\beta_{1} R^{2}+\beta_{2} R_{\mu \nu \rho \sigma} R^{\mu \nu \rho \sigma}+\beta_{3} R_{\mu \nu} R^{\mu \nu}\right)\right] .
$$

In particular for Gauss-Bonnet correction, $\beta_{1}=1, \beta_{2}=1, \beta_{3}=-4$. One can get rid of the $R i c c i^{2}$ and $S c a l a r^{2}$ terms by a field redefinition and therefore all physical quantities should depend on the coefficient $\beta_{2}$ only. Here we prefer to work with the generic case as it would be easier for us the get the results for pure Gauss-Bonnet combination at every step.

The background solution is given by [30],

$$
d s^{2}=f(r) d t^{2}+\frac{g(r)}{4 r^{3}} d r^{2}+\frac{1}{r} d \vec{x}^{2}
$$

where $f(r)$ and $g(r)$ are given by,

$$
f(r)=r-\frac{1}{r}-2 r\left(r^{2}-1\right) \beta_{2} \alpha^{\prime}
$$

and

$$
g(r)=\frac{r}{1-r^{2}}+\frac{2 r\left(10 \beta_{1}+\left(1-3 r^{2}\right) \beta_{2}+2 \beta_{3}\right) \alpha^{\prime}}{3\left(r^{2}-1\right)}
$$

This is the background metric corrected up to order $\alpha^{\prime}$. We have fixed the integration constant such that the boundary metric is Minkowskian and the horizon is located at $r=1$. The temperature of the black brane is given by,

$$
T=\frac{1}{\pi}+\frac{10 \beta_{1}-5 \beta_{2}+2 \beta_{3}}{3 \pi} \alpha^{\prime} .
$$

Similar to the $W_{e y l}^{4}$ case, we can write the following effective action for this model,

$$
S_{\mathrm{eff}}=\frac{1}{16 \pi G_{5}} \int \frac{d^{4} k}{(2 \pi)^{4}} d r\left[\mathcal{A}_{1}^{G B}(r, k) \phi^{\prime}(r, k) \phi^{\prime}(r,-k)+\mathcal{A}_{0}^{G B}(r, k) \phi(r, k) \phi(r,-k)\right]
$$

where, $\mathcal{A}_{1}^{G B}$ and $\mathcal{A}_{0}^{G B}$ are given in appendix D. Now, it is straightforward to write the flow equation (2.15) corresponding to this case,

$$
\partial_{r} \bar{\chi}^{\mathrm{GB}}\left(k_{\mu}, r\right)=i \omega \sqrt{-\frac{g_{r r}}{g_{t t}}}\left[\frac{\bar{\chi}^{\mathrm{GB}}\left(k_{\mu}, r\right)^{2}}{\Sigma^{\mathrm{GB}}(r, k)}-\frac{\Upsilon^{\mathrm{GB}}(r, k)}{\omega^{2}}\right],
$$

where we define

$$
\begin{aligned}
& \Sigma^{\mathrm{GB}}(r, k)=-2 \mathcal{A}_{1}^{\mathrm{GB}}\left(r, k_{\mu}\right) \sqrt{-\frac{g_{r r}}{g_{t t}}} \\
& \Upsilon^{\mathrm{GB}}(r, k)=2 \mathcal{A}_{0}^{\mathrm{GB}}\left(r, k_{\mu}\right) \sqrt{-\frac{g_{t t}}{g_{r r}}}
\end{aligned}
$$


Now, the boundary condition (2.18) takes the following form,

$$
\bar{\chi}^{G B}\left(k_{\mu}, 1\right)=\frac{1}{16 \pi G_{5}}\left[1+\left(\left(q^{2}-8\right) \beta_{3}-40 \beta_{1}\right) \alpha^{\prime}\right] .
$$

As mentioned earlier, we see that even in this case, the boundary condition depends on spatial momenta $q$ through the coefficient $\beta_{3}$. With this boundary condition, one can solve the flow equation (4.21) and the solution is given by,

$$
\begin{aligned}
i \omega \bar{\chi}^{G B}\left(k_{\mu}, 0\right) & =\frac{1}{16 \pi G_{5}}\left[-i\left(1-\left(40 \beta_{1}+8 \beta_{3}\right) \alpha^{\prime}\right) \omega\right. \\
& +\omega^{2}\left[\frac{1}{2}(1-\log 2)+\frac{\alpha^{\prime}}{6}\left(130 \beta_{1}(\log 2-1)-\beta_{2}(5 \log 2-2)+26 \beta_{3}(\log 2-1)\right)\right] \\
& \left.-\frac{q^{2}}{2}\left[1-\frac{1}{3}\left(130 \beta_{1}+25 \beta_{2}+26 \beta_{3}\right) \alpha^{\prime}\right]\right]+\mathcal{O}\left(q \omega^{2}, \omega q^{2}, q^{3}, \omega^{3}\right)
\end{aligned}
$$

From this expression we get the following transport coefficients,

$$
\eta=\frac{1}{16 \pi G_{5}}\left(1-8\left(5 \beta_{1}+\beta_{3}\right) \alpha^{\prime}\right)+\mathcal{O}\left(\alpha^{2}\right)
$$

This matches with results in $[40,42,43]$. The higher order coefficients are,

$$
\begin{aligned}
\kappa & =\frac{\eta}{\pi T}\left(1-10 \beta_{2} \alpha^{\prime}\right)+\mathcal{O}\left(\alpha^{\prime 2}\right) \\
\tau_{\pi} T & =\frac{2-\ln 2}{2 \pi}-\frac{11 \beta_{2}}{2 \pi} \alpha^{\prime}+\mathcal{O}\left(\alpha^{\prime 2}\right) .
\end{aligned}
$$

As we can see, the physical quantities $\eta / s, \kappa, \tau_{\pi} T$ only depend on the coefficient $\beta_{2}$. In particular to Gauss-Bonnet combination, the corrections are,

$$
\begin{aligned}
\kappa & =\frac{\eta}{\pi T}\left(1-10 \alpha^{\prime}\right)+\mathcal{O}\left(\alpha^{2}\right) \\
\tau_{\pi} T & =\frac{2-\ln 2}{2 \pi}-\frac{11}{2 \pi} \alpha^{\prime}+\mathcal{O}\left(\alpha^{\prime 2}\right) .
\end{aligned}
$$

(4.27) and (4.28) are new results of this paper.

\subsubsection{Exact result for Gauss-Bonnet black hole}

As we have done the above computation perturbatively, the above expressions are valid only at order $\alpha^{\prime}$. But, one can consider the Gauss-Bonnet term exactly in coupling. For pure Gauss-Bonnet combination the equations of motions remain second order differential equations and hence it is easy to solve them exactly to find the background space-time. We solve the flow equation in this background exactly in coupling constant, and find the exact expressions for relaxation time $\tau_{\pi}$ and $\kappa$. In this section we briefly outline the result.

The action and the solution is given by,

$$
\begin{aligned}
\mathcal{I}_{G B} & =\frac{1}{16 \pi G_{5}} \int d^{5} x \sqrt{-g}\left[R+12+\frac{\lambda_{g b}}{2}\left(R^{2}+R_{\mu \nu \rho \sigma} R^{\mu \nu \rho \sigma}-4 R_{\mu \nu} R^{\mu \nu}\right)\right] \\
d s^{2} & =r^{2}\left(-\frac{f(r)}{f_{\infty}} d t^{2}+d \vec{x}^{2}\right)+\frac{d r^{2}}{r^{2} f(r)}
\end{aligned}
$$


where,

$$
f_{ \pm}=\frac{1}{2 \lambda_{g b}}\left[1+ \pm \sqrt{1-4 \lambda_{g b}\left(1-\frac{r_{0}^{4}}{r^{4}}\right)}\right]
$$

and

$$
f_{\infty}=\lim _{r \rightarrow \infty} f(r)=\frac{1-\sqrt{1-4 \lambda_{g b}}}{2 \lambda_{g b}} .
$$

In this coordinate the boundary metric is $\eta$. We also consider only the ${ }^{\prime}{ }^{\prime}$ branch of $f_{ \pm}$which corresponds to a non-singular black hole solution with non-degenerate horizon.

The black hole temperature is given by,

$$
T=\frac{r_{0}}{\pi f_{\infty}} .
$$

With exact GB, the effective action for fluctuation has a canonical form. Therefore we derive the flow equation for the response function (as we did in section 2) and solving this equation we get,

$$
\begin{aligned}
\eta= & \frac{1}{16 \pi G_{5}}\left(1-4 \lambda_{g b}\right) \\
\kappa= & \frac{\eta}{\pi T} \frac{2 \lambda_{g b}\left(8 \lambda_{g b}-1\right)}{\left(1-\sqrt{1-4 \lambda_{g b}}\right)\left(4 \lambda_{g b}-1\right)} \\
\tau_{\pi} T= & \frac{1}{4 \pi\left(-1+4 \lambda_{g b}\right)}\left[-8 \lambda_{g b}^{2}+12 \sqrt{1-4 \lambda_{g b}} \lambda_{g b}\right. \\
& +10 \lambda_{g b}-2 \sqrt{1-4 \lambda_{g b}}-4 \log (2) \lambda_{g b} \\
& +\left(1-4 \lambda_{g b}\right) \log \left(-4 \lambda_{g b}+\sqrt{1-4 \lambda_{g b}}+1\right) \\
& \left.+\left(4 \lambda_{g b}-1\right) \log \left(1-4 \lambda_{g b}\right)-2+\log (2)\right] .
\end{aligned}
$$

One can easily check that up to first order in $\lambda_{g b}$, the results in (4.33) reduces to the one in (4.28). In [44] the authors obtained the relation between second order transport coefficients and $\lambda_{g b}$ numerically, however we are able to present the result exactly.

As we have mentioned in introduction that the flow equation is a first order non-linear differential equation but one can reduce this equation to a second order linear differential equation. This second order differential equation is related to the equation of motion for transverse graviton (gauge invariant excitations). Therefore we can use this equation to study causality violation in Gauss-Bonnet gravity. In [44, 45] it was found that to preserve causality of a conformal fluid there exists a bound on second order transport coefficients,

$$
\tau_{\pi} T-2 \frac{\eta}{s} \geq 0
$$

In Fig 3 we plot $\tau_{\pi} T-2 \frac{\eta}{s}$ for our result and find the following bound on $\lambda_{g b}$ which is in agreement with [44].

$$
-0.711 \leq \lambda_{g b} \leq 0.113
$$




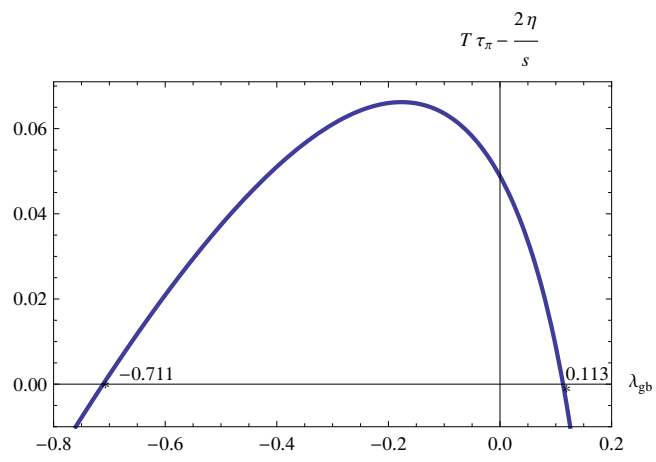

Figure 3. Bound on $\lambda_{g b}$.

\section{$5 \quad$ Flow equation for charged black holes}

Electrically charged black holes in five dimensions have drawn a lot of interests in the context of AdS/CFT. The electric charge of these black holes are mapped to the global R-charge of the dual field theory. Because of the presence of the electric charges, the thermodynamics and the phase structure of these black holes are rather complicated and also interesting at the same time. There have been a lot of study of thermodynamics and phase transitions of these charged black hole with different horizon topologies (see [46-50] and references therein).

The goal of the present section is to apply the AdS/CFT correspondence to understand how non-vanishing chemical potentials effect the hydrodynamic behavior of strongly coupled gauge theories. We study the second order hydrodynamics in two cases: (a) Generic R-charge black holes and (b) Charge black holes in higher-derivative gravity.

\section{$5.1 \quad$ R-charged black holes}

We consider a conformal field theory with conserved charge (density) in addition to energy and momentum. This is especially an interesting extension of the hydrodynamics of the uncharged fluids.

The second order hydrodynamics of charged fluid has been studied in $[51,52]$. They consider Reissner-Nordstrom black hole in five dimensions and found the effect of chemical potential on second order transport coefficients in some limits of chemical potential. However, we consider generic $R$-charged black holes with three (unequal) charges (chemical potentials) and find the exact expressions for second order transport coefficients in presence of three chemical potentials. As we have mentioned in the introduction that solving the flow equation (of retarded Green's function of energy momentum tensor) we can only find two second order transport coefficients whereas in [51,52] all other second order transport coefficients have been reported.

We consider R-charged black holes in five dimensions. A consistent truncation of $\mathcal{N}=$ $8, D=5$ gauged supergravity with $\mathrm{SO}(6)$ Yang-Mills gauge group, which can be obtained by $S^{5}$ reduction of type $I I B$ supergravity, gives rise to $\mathcal{N}=2, D=5$ gauge supergravity with $\mathrm{U}(1)^{3}$ gauge group. The same theory can also be obtained by compactifying eleven 
dimensional supergravity, low energy theory of $M$ theory, on a Calabi-Yau three folds. The bosonic part of the action of $\mathcal{N}=2, D=5$ gauged supergravity is given by [46-50]. We follow the notations of [53].

$$
\begin{aligned}
\mathcal{I}_{\text {sugra }}= & \frac{1}{16 \pi G_{5}} \int d^{5} x \sqrt{-g}\left[R+V(X)-\frac{1}{2} G_{I J}(X) F_{\mu \nu}^{I} F^{\mu \nu J}-G_{I J}(X) \partial_{\mu} X_{I} \partial^{\mu} X_{J}\right] \\
& +\frac{\frac{\zeta}{3}}{16 \pi G_{5}} \int d^{5} x \epsilon^{\mu \nu \rho \sigma \gamma} A_{\mu} F_{\nu \rho} F_{\sigma \gamma}
\end{aligned}
$$

where, $X^{I}$ 's are three real scalar fields, subject to the constraint $X^{1} X^{2} X^{3}=1 . F^{I}$ 's, the field strengths of three Abelian gauge fields $(\mathrm{I}, \mathrm{J}=1,2,3)$ and the scalar potential $V(X)$ are given by,

$$
\begin{aligned}
F_{\mu \nu}^{I} & =2 \partial_{[\mu} A_{\nu]}^{I} \\
G_{I J} & =\frac{1}{2} \operatorname{diag}\left[\left(\mathrm{X}^{1}\right)^{-2},\left(\mathrm{X}^{2}\right)^{-2},\left(\mathrm{X}^{3}\right)^{-2}\right] \\
V(X) & =2 \sum_{I} \frac{1}{X_{I}}
\end{aligned}
$$

The three-charge non-extremal STU solution is specified by the following background values of the metric

$$
\begin{aligned}
d s^{2} & =-\mathcal{H}^{-2 / 3} f_{k} d t^{2}+\mathcal{H}^{1 / 3}\left(f_{k}^{-1} d r^{2}+r^{2} d \Omega_{3, k}^{2}\right), \\
f_{k} & =k-\frac{m_{k}}{r^{2}}+r^{2} \mathcal{H}, \quad H_{i}=1+\frac{q_{i}}{r^{2}}, \\
\mathcal{H} & =H_{1} H_{2} H_{3}
\end{aligned}
$$

as well as the scalar and the gauge fields

$$
X^{i}=\frac{\mathcal{H}^{1 / 3}}{H_{i}}, \quad A_{t}^{i}=\sqrt{\frac{k q_{i}+m_{k}}{q_{i}}}\left(1-H_{i}^{-1}\right) .
$$

The parameter $k$ determines the spatial curvature of $d \Omega_{3, k}^{2}: k=1$ corresponds to the metric on the three-sphere of unit radius, $k=0$ - to the metric on $R^{3}$. Hydrodynamic approximation is valid only in the case of a translational-invariant horizon, in our case we set $k=0$ and

$$
d \Omega_{3,0}^{2} \rightarrow\left(d x^{2}+d y^{2}+d z^{2}\right) .
$$

Replacing the radial coordinate $r \rightarrow r_{0} / \sqrt{r}$, where $r_{0}$ is the largest root of the equation $f(r)=0$, the background solution in this new coordinate is given by,

$$
\begin{aligned}
d s_{5}^{2} & =-\mathcal{H}^{-2 / 3} \frac{\left(\pi T_{0}\right)^{2}}{r} f d t^{2}+\mathcal{H}^{1 / 3} \frac{1}{4 f r^{2}} d r^{2}+\mathcal{H}^{1 / 3} \frac{\left(\pi T_{0}\right)^{2}}{r}\left(d x^{2}+d y^{2}+d z^{2}\right), \\
f(r) & =\mathcal{H}(r)-r^{2} \prod_{i=1}^{3}\left(1+\kappa_{i}\right), \quad H_{i}=1+\kappa_{i} r \\
\kappa_{i} & \equiv \frac{q_{i}}{r_{0}^{2}}
\end{aligned}
$$


where $\kappa_{i}^{\prime} s$ are chemical potentials and

$$
T_{0}=r_{0} / \pi
$$

The scalar fields and the gauge fields are given by

$$
X^{i}=\frac{\mathcal{H}^{1 / 3}}{H_{i}(r)}, \quad A_{t}^{i}=\frac{\tilde{\kappa}_{i} \sqrt{2} r}{L H_{i}(r)}
$$

where,

$$
\tilde{\kappa}_{i}=\sqrt{q_{i}} \prod_{i=1}^{3}\left(1+\kappa_{i}\right)^{1 / 2} .
$$

The Hawking temperature of the background (5.5) is given by

$$
T_{H}=\frac{2+\kappa_{1}+\kappa_{2}+\kappa_{3}-\kappa_{1} \kappa_{2} \kappa_{3}}{2 \sqrt{\left(1+\kappa_{1}\right)\left(1+\kappa_{2}\right)\left(1+\kappa_{3}\right)}} T_{0} .
$$

We perturb the $x y$ component of background metric and the action for transverse graviton is,

$$
S_{\text {eff }}=\frac{1}{16 \pi G_{5}} \int \frac{d^{4} k}{(2 \pi)^{4}} d r\left[\mathcal{A}_{1}^{Q}(r, k) \phi^{\prime}(r, k) \phi^{\prime}(r,-k)+\mathcal{A}_{0}^{Q}(r, k) \phi(r, k) \phi(r,-k)\right]
$$

where,

$$
\mathcal{A}_{1}^{\mathrm{Q}}=-\frac{r_{0}^{4} f(r)}{r}
$$

and

$$
\mathcal{A}_{0}^{\mathrm{Q}}=\frac{r_{0}^{2}}{4 r^{2}}\left(\frac{H_{1} H_{2} H_{3}}{f(r)}-q^{2}\right)
$$

Therefore the flow equation takes the form,

$$
\partial_{r} \bar{\chi}^{\mathrm{Q}}\left(k_{\mu}, r\right)=i \omega \sqrt{-\frac{g_{r r}}{g_{t t}}}\left[\frac{\bar{\chi}^{\mathrm{Q}}\left(k_{\mu}, r\right)^{2}}{\Sigma^{\mathrm{Q}}(r, k)}-\frac{\Upsilon^{\mathrm{Q}}(r, k)}{\omega^{2}}\right] .
$$

Solving this equation perturbatively in $\omega$ and $q$ we get

$$
\begin{aligned}
\bar{\chi}^{\mathrm{Q}}\left(k_{\mu}, r\right)= & -\frac{r_{0}^{3} \prod_{i}\left(1+\kappa_{i}\right)^{1 / 2}}{16 \pi G_{5}}+\frac{i r_{0}^{2}}{2 \omega} \frac{\left(q^{2}-\omega^{2}\right)}{16 \pi G_{5}}\left(1-\frac{1}{r}\right) \\
& +\frac{i \omega r_{0}^{2} \prod_{i}\left(1+\kappa_{i}\right)}{16 \pi G_{5} \sqrt{4 P_{\kappa}+\left(1+S_{\kappa}\right)^{2}}} \\
& \left(\frac{1}{2} \ln \left[\frac{1+S_{\kappa}-2 P_{\kappa}-\sqrt{4 P_{\kappa}+\left(1+S_{\kappa}\right)^{2}}}{1+S_{\kappa}-2 r S_{\kappa}-\sqrt{4 P_{\kappa}+\left(1+S_{\kappa}\right)^{2}}}\right]\right. \\
& \left.+\frac{1}{2} \ln \left[\frac{1+S_{\kappa}-2 r P_{\kappa}+\sqrt{4 P_{\kappa}+\left(1+S_{\kappa}\right)^{2}}}{1+S_{\kappa}-2 S_{\kappa}+\sqrt{4 P_{\kappa}+\left(1+S_{\kappa}\right)^{2}}}\right]\right) \\
& +\mathcal{O}\left(q \omega^{2}, \omega q^{2}, q^{3}, \omega^{3}\right)
\end{aligned}
$$


where,

$$
\begin{aligned}
& S_{\kappa}=\sum_{i} \kappa_{i} \\
& P_{\kappa}=\prod_{i} \kappa_{i} .
\end{aligned}
$$

Computing the response function at the boundary (throwing away the divergent piece) we get the following transport coefficients,

$$
\eta=\frac{r_{0}^{3}}{16 \pi G_{5}} \prod_{i}\left(1+\kappa_{i}\right)^{1 / 2}
$$

and

$$
\begin{aligned}
\kappa & =\frac{\eta}{\pi T} \frac{1+S_{\kappa} / 2-P_{\kappa} / 2}{\prod_{i}\left(1+\kappa_{i}\right)} \\
\tau_{\pi} T & =\frac{2+S_{\kappa}-P_{\kappa}}{4 \pi \prod_{i}\left(1+\kappa_{i}\right)}\left[2-\frac{\prod_{i}\left(1+\kappa_{i}\right)}{\sqrt{4 P_{\kappa}+\left(1+S_{\kappa}\right)^{2}}} \ln \left(\frac{3+S_{\kappa}+\sqrt{4 P_{\kappa}+\left(1+S_{\kappa}\right)^{2}}}{3+S_{\kappa}-\sqrt{4 P_{\kappa}+\left(1+S_{\kappa}\right)^{2}}}\right)\right] .
\end{aligned}
$$

These are the new results in this paper. It is easy to check that for $\kappa_{i} \rightarrow 0$ limit we recover the results in section 2 .

To complete the discussion on the second order transport coefficients for $R$-charged black holes one should find the flow of Green's functions for two point correlation functions of $R$-currents. As we will mention in section 6 that in presence of finite charges (or chemical potentials) it is very hard to solve the Riccati equation even perturbatively in $\omega$ and $q$. We find it very difficult to get any analytic solution for $R$-current Green's function. However we consider a simple model in section 6 and study the flow of $R$-current Green's function numerically.

\subsection{Charged black holes in higher derivative gravity}

In this section, we will study five-dimensional gravity in presence of a negative cosmological constant and coupled to U(1) gauge field. The model has been studied in [33, 54, 55], the action is given as,

$$
\begin{aligned}
S= & \frac{1}{16 \pi G_{5}} \int d^{5} x \sqrt{-g}\left[R+12-\frac{1}{4} F^{2}+\frac{\zeta}{3} \epsilon^{a b c d e} A_{a} F_{b c} F_{d e}\right. \\
& \left.+\alpha^{\prime}\left(c_{1} R_{a b c d} R^{a b c d}+c_{2} R_{a b c d} F^{a b} F^{c d}+c_{3}\left(F^{2}\right)^{2}+c_{4} F^{4}+c_{5} \epsilon^{a b c d e} A_{a} R_{b c f g} R_{d e}^{f g}\right)\right] .
\end{aligned}
$$

Here, $F^{2}=F_{a b} F^{a b}, F^{4}=F_{a b} F^{b c} F_{c d} F^{d a}$, and the AdS radius is set to unity. The action includes the Chern-Simon term and also a generic set of four derivative terms. All the four derivative terms will be treated perturbatively in our computation and here $\alpha^{\prime} \ll 1$ is the perturbation parameter. In [33], using field-redefinition, the authors have shown that, within perturbative approach, this is the most generic four derivative action that one can 
write down. In this section, we will closely follow their work. The background metric and the gauge field in presence of these higher derivative terms have the following form,

$$
\begin{aligned}
d s^{2} & =-r^{2} f(r) d t^{2}+\frac{1}{r^{2} g(r)} d r^{2}+r^{2}\left(d x^{2}+d y^{2}+d z^{2}\right), \\
A & =h(r) d t
\end{aligned}
$$

where,

$$
\begin{aligned}
& f(r)=f_{0}(r)\left(1+\alpha^{\prime} F(r)\right), \\
& g(r)=f_{0}(r)\left(1+\alpha^{\prime}(F(r)+G(r))\right), \\
& h(r)=h_{0}(r)+\alpha^{\prime} H(r) .
\end{aligned}
$$

Here $f_{0}(r), g_{0}(r)$ and $h_{0}(r)$ are the solution of the background in absence of the higherderivative terms in the action and they are given as,

$$
\begin{aligned}
& f_{0}=g_{0}=\left(1-\frac{r_{0}^{2}}{r^{2}}\right)\left(1+\frac{r_{0}^{2}}{r^{2}}-\frac{Q^{2}}{r_{0}^{2} r^{4}}\right), \\
& h_{0}=\sqrt{3} Q\left(\frac{1}{r_{0}^{2}}-\frac{1}{r^{2}}\right) .
\end{aligned}
$$

Here, $Q$ is related to the physical charge of the system and $r_{0}$ is the position of the horizon. From (5.21), it is clear that even in presence of the higher-derivative terms, the horizon remains at $r_{0}$. The higher-derivative corrections to this background are given by the functions $F(r), G(r)$ and $H(r)$. The form of these functions are given in [33]. We would not write those expressions and refer the reader to that paper.

Using the flow equation, we will study the higher order transport coefficient of the plasma theory dual to this gravity model. For this, we will write the effective action for the metric fluctuation in (2.3), as we have done in previous sections,

$$
S_{\mathrm{eff}}=\frac{1}{16 \pi G_{5}} \int \frac{d^{4} k}{(2 \pi)^{4}} d r\left[\mathcal{A}_{1}^{C B}(r, k) \phi^{\prime}(r, k) \phi^{\prime}(r,-k)+\mathcal{A}_{0}^{C B}(r, k) \phi(r, k) \phi(r,-k)\right] .
$$

$\mathcal{A}_{1}^{C B}$ and $\mathcal{A}_{0}^{C B}$ are given in appendix E. The corresponding flow equation (2.15) for this case (with the coefficients $\mathcal{A}_{1}^{C B}$ and $\mathcal{A}_{0}^{C B}$ ) is,

$$
\partial_{r} \bar{\chi}^{\mathrm{CB}}\left(k_{\mu}, r\right)=i \omega \sqrt{-\frac{g_{r r}}{g_{t t}}}\left[\frac{\bar{\chi}^{\mathrm{CB}}\left(k_{\mu}, r\right)^{2}}{\Sigma^{\mathrm{CB}}(r, k)}-\frac{\Upsilon^{\mathrm{CB}}(r, k)}{\omega^{2}}\right],
$$

where we define

$$
\begin{aligned}
& \Sigma^{\mathrm{CB}}(r, k)=-2 \mathcal{A}_{1}^{\mathrm{CB}}\left(r, k_{\mu}\right) \sqrt{-\frac{g_{r r}}{g_{t t}}} \\
& \Upsilon^{\mathrm{CB}}(r, k)=2 \mathcal{A}_{0}^{\mathrm{CB}}\left(r, k_{\mu}\right) \sqrt{-\frac{g_{t t}}{g_{r r}}} .
\end{aligned}
$$

We solve this flow equation to find the effect of higher derivative terms and chemical potential (or charge) on transport coefficient. Here, we present our result for small $Q$ only, though it is possible to find the results for any $Q$. 
The boundary condition (2.18) will take the following form, ${ }^{18}$

$$
\bar{\chi}\left(k_{\mu}, r_{0}\right)=\frac{r_{0}^{3}}{16 \pi G_{5}}\left[1-\alpha^{\prime} \frac{24 c_{1} Q^{2}}{r_{0}^{6}}\right] .
$$

In this case, the horizon value of the response function is independent of momenta. With this boundary condition, we can solve the flow equation (5.24), and the solution is given by,

$$
\begin{aligned}
i \omega \bar{\chi}\left(k_{\mu}, \infty\right)= & i \omega \frac{r_{0}^{3}}{16 \pi G_{5}}\left[1-\alpha^{\prime} \frac{24 c_{1} Q^{2}}{r_{0}^{6}}\right] \\
& -\omega^{2} \frac{r_{0}^{2}}{32 \pi G_{5}}\left[(1-\ln 2)-\frac{Q^{2}}{2 r_{0}^{6}}(3-\ln 2)\right. \\
& \left.+\frac{\alpha^{\prime}}{6}\left(c_{1}(2-5 \ln 2)-\frac{Q^{2}}{2 r_{0}^{6}}\left(c_{1}(35-58 \ln 2)-48 c_{2}(2-\ln 2)\right)\right)\right] \\
& +\frac{q^{2} r_{0}^{2}}{32 \pi G_{5}}\left[1-\frac{\alpha^{\prime}}{3}\left(25 c_{1}+\frac{Q^{2}}{r_{0}^{6}}\left(32 c_{1}+24 c_{2}\right)\right)\right] \\
& +\mathcal{O}\left(q \omega^{2}, \omega q^{2}, q^{3}, \omega^{3}\right)+\mathcal{O}\left(Q^{4}\right) .
\end{aligned}
$$

It is easy to read off the transport coefficients from this expression as,

$$
\begin{aligned}
\eta= & \frac{r_{0}^{3}}{16 \pi G_{5}}\left[1-\alpha^{\prime} \frac{24 c_{1} Q^{2}}{r_{0}^{6}}\right]+\mathcal{O}\left(Q^{4}\right) \\
\kappa= & \frac{\eta}{\pi T}\left[\left(1-\frac{Q^{2}}{2 r_{0}^{6}}\right)-\alpha^{\prime}\left(10 c_{1}-\frac{Q^{2}}{3 r_{0}^{6}}\left(37 c_{1}-48 c_{2}\right)\right)\right]+\mathcal{O}\left(Q^{4}\right) \\
\tau_{\pi} T= & \frac{2-\ln 2}{2 \pi}-\frac{Q^{2}(5-3 \ln 2)}{4 \pi r_{0}^{6}} \\
& +\alpha^{\prime}\left[-\frac{11 c_{1}}{2 \pi}+\frac{Q^{2}}{4 \pi r_{0}^{6}}\left(-16 c_{2}+5 c_{1}(11-4 \ln 2)\right)\right]+\mathcal{O}\left(Q^{4}\right)
\end{aligned}
$$

where, the temperature $T$ of the system is given by,

$$
T=\frac{r_{0}}{\pi}\left[\left(1-\frac{Q^{2}}{2 r_{0}^{6}}\right)-\frac{\alpha^{\prime}}{3}\left(5 c_{1}+\frac{Q^{2}}{2 r_{0}^{6}}\left(31 c_{1}+48 c_{2}\right)\right)\right]+\mathcal{O}\left(Q^{4}\right) .
$$

We see that the first order as well as the second order transport coefficients coming from retarded Green's function of energy momentum tensor ${ }^{19}$ only depends on two coefficients $c_{1}, c_{2}$. This feature was observed in [33] for entropy density $s$ and first-order transport coefficients $\eta$. The coefficients $c_{3}, c_{4}$, which parameterize couplings in the four point function of the dual U(1) current does not play any role in these hydrodynamic coefficients. They

\footnotetext{
${ }^{18}$ Note that we are working in a different coordinate where $r \rightarrow \infty$ is the boundary, therefore we choose the positive branch of the boundary condition (2.18).

${ }^{19}$ It would be interesting to study the flow of retarded Green's function for boundary $R$-current. We found it to be difficult to get any analytical solution for response function in presence of finite chemical potential and higher derivative terms. However, it would be nice to know the higher derivative corrections to other second order transport coefficients appear in $R$ current [51, 52].
} 
should be important for the computation of conductivity, which comes from the Green's function of the boundary $R$-current. Two other coefficients $\zeta, c_{5}$ also do not appear in the expressions. One can find a magnetic brane solution of the action (5.19) like [56]. In that case it would be interesting to find the effect of magnetic field on transport coefficients.

\section{Flow of retarded Green's function of boundary $R$ current}

Finally, in this section, we study the flow of retarded Green's function of boundary $R$-current,

$$
G_{i, j}^{R}(k)=-i \int d t d^{3} x e^{i k \cdot x}\left\langle\left[J_{i}(x), J_{j}(0)\right]\right\rangle
$$

where $J_{\mu}(x)$ is the $C F T$ current dual to a bulk gauge field $A_{\mu}$.

In hydrodynamic approximation one can express the current in powers of boundary derivatives. Up to first order in derivative expansion (about equilibrium state) it has the following form,

$$
J_{\nu}=-\tilde{\kappa} P_{\nu}^{\alpha} \partial_{\alpha} \frac{\mu}{T}+\Omega l_{\nu}+\mathcal{O}\left(\partial^{2}\right)
$$

where, $\tilde{\kappa}$ and $\Omega$ are two first order transport coefficients, $\mu$ is chemical potential, $T$ is temperature and

$$
\begin{aligned}
P_{\mu \nu} & =u_{\mu} u_{\nu}+\eta_{\mu \nu} \\
l_{\mu} & =\epsilon_{\mu}^{\alpha \beta \gamma} u_{\alpha} \partial_{\beta} u_{\gamma} .
\end{aligned}
$$

The expression of $J_{\mu}$ up to second order in derivative expansion can be found in [51, 52]. From conformal invariance of the theory it is possible to write all possible second order transport coefficients appearing in the expression of $J_{\mu}$. However, like energy momentum tensor, from the expression of retarded Green's function it is not possible to compute all the transport coefficients that appear in different order of derivative expansion.

In this section we study the flow equation of retarded Green's function of boundary $R$ current. Unfortunately we find it difficult to solve the flow equation analytically to extract any transport coefficient. We present the flow equation for retarded Green's function of $R$-currents in presence of generic higher derivative terms in bulk Lagrangian and some numerical results.

We start with Einstein-Maxwell action

$$
S=\frac{1}{16 \pi G_{5}} \int d^{5} x \sqrt{-g}\left(R+12-\frac{1}{4} F^{2}\right) .
$$

Background solution is given by equation (5.20) with $\alpha^{\prime}=0$. The temperature of the black hole is given by,

$$
T=\frac{r_{0}}{\pi}\left(1-\frac{Q^{2}}{2 r_{0}^{6}}\right)
$$

and the chemical potential is,

$$
\mu=\frac{\sqrt{3} Q}{r_{0}^{2}} .
$$


For technical advantage we write the metric and gauge field in a different coordinate. We change the radial coordinate $r \rightarrow \frac{r_{0}}{\sqrt{r}}$. In this coordinate the metric and gauge field are given by,

$$
\begin{aligned}
d s^{2} & =-\frac{r_{0}^{2} \mathrm{U}(r)}{r} d t^{2}+\frac{d r^{2}}{4 r^{2} \mathrm{U}(r)}+\frac{r_{0}^{2}}{r}\left(d \vec{x}^{2}\right) \\
A_{t}(r) & =E(r)
\end{aligned}
$$

where,

$$
\begin{aligned}
& \mathrm{U}(r)=(1-r)\left(1+r-\frac{Q^{2} r^{2}}{r_{0}^{6}}\right) \\
& E(r)=\frac{\sqrt{3} Q}{r_{0}^{2}}(1-r) .
\end{aligned}
$$

We turn on small fluctuations for $x$ component of gauge fields. Since the $A_{t}$ component of the bulk vector is non-vanishing in this background, the perturbations $A_{x}$ can couple to the $t x$ component of graviton. Therefore we also need to consider small metric fluctuations for component $g_{t x}$. Writing them in momentum space we get,

$$
\begin{aligned}
& A_{x}(r, x)=\int \frac{d^{4} k}{(2 \pi)^{4}} e^{i k \cdot x} A_{1}(r, k) \\
& g_{t}^{x}(r, x)=\int \frac{d^{4} k}{(2 \pi)^{4}} e^{i k \cdot x} \Phi(r, k) .
\end{aligned}
$$

However, there exists a constraint relation between $A_{x}$ and $g_{t x}$. We use this relation to replace $g_{t x}$ from the equation of motion of $A_{x}$.

The on-shell action for gauge field fluctuations is given by,

$$
S_{A}=\frac{1}{16 \pi G_{5}} \int \frac{d^{4} k}{(2 \pi)^{4}}\left[-r_{0}^{2} \mathrm{U}(r) A_{x}^{\prime 2}(r, k)+\left(\frac{\omega^{2}}{4 r \mathrm{U}(r)}-\frac{q^{2}}{4 r}\right) A_{x}^{2}\right] .
$$

and the current corresponding to $A_{x}$ fluctuation is,

$$
\begin{aligned}
J_{x}(r, k) & =\frac{\delta S_{A}}{\delta A_{x}^{\prime}(r, k)} \\
& =-2 r_{0}^{2} \mathrm{U}(r) A_{x}^{\prime}(r, k) .
\end{aligned}
$$

The equation of motion for $A_{x}(r, k)$ becomes,

$$
\begin{aligned}
\left(\mathrm{U}(r) A_{x}^{\prime}(r, k)\right)^{\prime} & =-\frac{1}{4 r_{0}^{2}}\left(\frac{\omega^{2}}{r \mathrm{U}(r)}-\frac{q^{2}}{r}\right) A_{x}(r, k)-E^{\prime}(r) \phi^{\prime}(r, k) \\
& =-\frac{1}{4 r_{0}^{2}}\left(\frac{\omega^{2}}{r \mathrm{U}(r)}-\frac{q^{2}}{r}\right) A_{x}(r, k)+\frac{r E^{\prime}(r)^{2}}{r_{0}^{2}} A_{x}(r, k)
\end{aligned}
$$

Using the constraint relation (coming from $r x$ component of Einstein equations),

$$
\phi^{\prime}(r, k)=-\frac{r E^{\prime}(r) A_{x}(r, k)}{r_{0}^{2}} .
$$




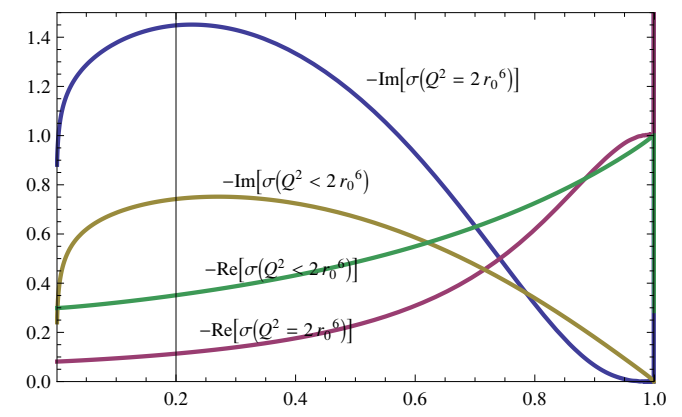

Figure 4. Flow of $\sigma$ for non-extremal and extremal black holes.

and the equations of motion one gets,

$$
J_{x}^{\prime}(r, k)=\frac{1}{2}\left(\frac{\omega^{2}}{r \mathrm{U}(r)}-\frac{q^{2}}{r}\right) A_{x}(r, k)-2 r E^{\prime}(r)^{2} A_{x}(r, k) .
$$

Next we define a response function

$$
\sigma(r, k)=\frac{J_{x}(r, k)}{i \omega A_{x}(r, k)}
$$

Taking the derivative of the response function with respect to $r$ and using the equation of motion we find the following flow equation,

$$
\sigma^{\prime}(r, k)=\frac{i \omega}{2 r_{0} \mathrm{U}(r)}\left[\sigma(r, k)^{2}-\left(\frac{r_{0}}{r}\left(1-\frac{q^{2} \mathrm{U}(r)}{\omega^{2}}\right)-\frac{4 r_{0} r E^{\prime}(r)^{2} \mathrm{U}(r)}{\omega^{2}}\right)\right] .
$$

The boundary condition which comes from the regularity of the response function at the horizon, demands

$$
\sigma(1, k)^{2}=1
$$

With this boundary condition one can integrate the nonlinear equation to find finite frequency response of boundary Green's function.

In presence of generic higher derivative terms in the Lagrangian the on-shell action for fluctuation $A_{x}$ may not have canonical form like (6.10). In that case one has to write an effective action for $A_{x}$ (like transverse graviton). The effective will have the same form as (6.10) only the coefficients will now depend on coupling constant of higher derivative terms.

We conclude this section by presenting some numerical solutions of the flow equation (6.16) for both non-extremal extremal black holes in figure 4.

\section{Acknowledgments}

We would like to thank Dileep Jatkar and Umut Gursoy for useful discussions. The work of NB is part of the research program of the Foundation for Fundamental Research on Matter (FOM), which is financially supported by the Netherlands Organization for Scientific Research (NWO). 


\section{A Second order transport coefficients from Kubo formula}

In this appendix we re-derive second order transport coefficients $\kappa$ and $\tau_{\pi}$ from usual Kubo approach. Let us now consider the action with solution given in section 2 ,

$$
S=\frac{1}{16 \pi G_{5}} \int d^{5} x \sqrt{-g}[R+12] .
$$

For well defined variation of this action we need to add a Gibbons-Hawking boundary term. Also, requiring the on-shell action being finite at boundary, we have to add counter-terms following usual approach of holographic renormalization. They are as follows:

$$
\begin{aligned}
S_{G H} & =\frac{1}{8 \pi G_{5}} \int d^{4} x \sqrt{-\gamma} \mathcal{K}, \\
S_{C T} & =\frac{1}{16 \pi G_{5}} \int d^{4} x \sqrt{-\gamma}\left[6+\frac{1}{2} \mathcal{R}\right]
\end{aligned}
$$

where $\gamma$ and $\mathcal{R}$ are boundary metric and Ricci scalar (constructed out of $\gamma$ ) respectively.

We consider the following metric perturbation,

$$
g_{x y}=g_{x y}^{(0)}+h_{x y}(r, x)=g_{x y}^{(0)}(1+\epsilon \Phi(r, x)),
$$

where $\epsilon$ is an order counting parameter. We are interested in quadratic on-shell action for this transverse graviton $\Phi(r, x)$. Let us define the Fourier transform,

$$
\phi(r, k)=\int \frac{d^{4} x}{(2 \pi)^{4}} e^{-i k \cdot x} \Phi(r, x),
$$

and $k=\{-\omega, \vec{k}\}$. Substituting this fluctuation in action (A.1), we get (B.1). ${ }^{20}$ Now, we rewrite this action (A.1) as equation of motion piece (which vanishes on-shell) and boundary term. ${ }^{21}$ Thus, on-shell $S, S_{G H}, S_{C T}$ become,

$$
\begin{aligned}
S & =\frac{1}{16 \pi G_{5}} \int_{r=\delta} \frac{d^{4} k}{(2 \pi)^{4}} L(\phi(r, k)), \\
S_{G H} & =\frac{1}{8 \pi G_{5}} \int_{r=\delta} \frac{d^{4} k}{(2 \pi)^{4}} L_{G H}(\phi(r, k)), \\
S_{C T} & =\frac{1}{16 \pi G_{5}} \int_{r=\delta} \frac{d^{4} k}{(2 \pi)^{4}} L_{C T}(\phi(r, k)),
\end{aligned}
$$

where we define,

$$
\begin{aligned}
L(\phi(r, k)) & =a 2(r) \phi^{\prime}(r, k) \phi^{*}(r, k)+\frac{a 4(r)-a 5^{\prime}(r)}{2} \phi(r, k) \phi^{*}(r, k), \\
L_{G H}(\phi(r, k)) & =2(g 1) \phi(r, k) \phi^{*}(r, k)+2(g 2) \phi^{\prime}(r, k) \phi^{*}(r, k), \\
L_{C T}(\phi(r, k)) & =\left(c_{0}+c_{1} \omega^{2}+c_{2} q^{2}\right) \phi(r, k) \phi^{*}(r, k) .
\end{aligned}
$$

\footnotetext{
${ }^{20} a_{3}, a_{6}$ are zero here.

${ }^{21}$ We ignore contribution from horizon as [5].
} 
The coefficients $a_{2}, a_{4}, a_{5}$ are given in (B.2) and other coefficients are,

$$
\begin{aligned}
g_{1} & =\frac{2-r^{2}}{r^{2}}, & g_{2} & =-2 \frac{1-r^{2}}{r} \\
c_{0} & =-3 \frac{\sqrt{1-r^{2}}}{r^{2}}, & c_{1} & =\frac{1}{4 r \sqrt{1-r^{2}}} \\
c_{2} & =-\frac{\sqrt{1-r^{2}}}{4 r} & &
\end{aligned}
$$

The retarded Green's function is defined at asymptotic infinity as,

$$
G^{R}(k)=2 \lim _{r \rightarrow 0} \frac{\left.\left(L+L_{G H}+L_{C T}\right)\right|_{\text {on-shell }}}{\phi_{0}(k) \phi_{0}(-k)} .
$$

Here, $\phi_{0}(k)$ is the boundary value of the fluctuation (A.4). The retarded Green's function $G^{R}$ is a function of boundary momenta $k_{\mu}=(\omega, 0,0, q)$. Now the leading action and the Gibbons-Hawking action get divergences from $\phi^{\prime}(r, k) \phi^{*}(r, k)$ and $\phi(r) \phi^{*}(r, k)$ parts. Both these divergences get canceled by the counter-term action which is always proportional to only $\phi(r) \phi^{*}(r, k)$. In this case of leading Einstein's gravity, it is even more simplified. Divergences coming from $\phi(r) \phi^{*}(r, k)$ piece of leading and Gibbons-Hawking action gets canceled by momentum independent piece of Counter-term action. It turns out that there is a cancellation among the corresponding coefficients as,

$$
\lim _{r \rightarrow 0}\left(\frac{1}{2}\left(a 4(r)-a 5^{\prime}(r)\right)+2 g 1+c_{0}\right)=\frac{1}{2},
$$

i.e. the final contribution from $\phi(r) \phi^{*}(r, k)$ piece is only a finite number $\frac{1}{2}$. As the graviton fluctuation $\phi(r, \omega, \vec{k})=\phi_{0}(1+F(r, \omega, \vec{k}))$ and moreover $\lim _{r \rightarrow 0} F(r, \omega, \vec{k})=0$, we see that the $\phi(r) \phi^{*}(r)$ term above only contribute to pressure (the $\omega$ independent piece of $G^{R}$ ). It would never contribute to any transport coefficient.

Also, the divergences coming from $\phi^{\prime}(r, k) \phi^{*}(r, k)$ piece of original and GibbonsHawking action, get canceled with the piece of the counter-term proportional to $\omega^{2}, q^{2}$ ( $c_{1}$ and $c_{2}$ are purely divergent at boundary). Here, the situation is more subtle, as there is no cancellation among the coefficients. One actually needs to put the solution of $\phi(r, \omega, \vec{k})$ to see the cancellation.

The overall lesson from this detailed analysis is that counter-term only cancel the UV divergences in usual holographic renormalization process and at most contribute to pressure of the boundary plasma. It has no effects on any transport coefficients. In [10], the author have computed second order transport coefficients for the plasmas dual to leading Einstein's gravity following this usual approach. The results are as follows,

$$
\tau_{\pi}=\frac{2-\ln 2}{2 \pi T}, \quad \kappa=\frac{\eta}{\pi T} .
$$

These results match with the one we obtained in (2.28) by solving the flow equations. 


\section{B Equivalence of boundary terms}

In this appendix we will show explicitly why the transport coefficients computed from the original action and the effective action are same, even for any higher derivative theory. It was already noticed [29], that the two would give same first-order transport coefficient $\eta$ with a suitable choice of the overall normalization constant. Here, we show that, not just the first order transport coefficients, rather any higher order transport coefficients computed from the original action and the effective action are same.

We consider a general class of action for $\phi$ which appears when the higher derivative terms are made of different contraction of Ricci tensor, Riemann tensor, Weyl tensor, Ricci scalar etc. or their different powers. Since, all these tensors involve two derivatives of metric they can only have terms like $\partial_{a} \partial_{b} \Phi(r, x)$ and its lower derivatives. Therefor the most generic quadratic (in $\Phi(r, x)$, in linear response theory) action for this kind of higher derivative gravity has the following form (in momentum space) ${ }^{22}$

$$
\begin{array}{r}
S=\frac{1}{16 \pi G_{5}} \int \frac{d^{4} k}{(2 \pi)^{4}} d r\left[a 1(r) \phi(r)^{2}+a 2(r) \phi^{\prime}(r)^{2}+a 4(r) \phi(r) \phi^{\prime}(r)\right. \\
\left.+\alpha^{\prime} a 6(r) \phi^{\prime \prime}(r) \phi^{\prime}(r)+\alpha^{\prime} a 3(r) \phi^{\prime \prime}(r)^{2}+a 5(r) \phi(r) \phi^{\prime \prime}(r)\right]
\end{array}
$$

where,

$$
\begin{aligned}
& a 1(r)=\frac{-8 r^{2}+\omega^{2} r+8}{4 r^{3}-4 r^{5}}+\alpha^{\prime} f 2(r) \\
& a 2(r)=-3 r+\frac{3}{r}+\alpha^{\prime} h 2(r) \\
& a 4(r)=-\frac{6}{r^{2}}-2+\alpha^{\prime} g 2(r) \\
& a 5(r)=-4 r+\frac{4}{r}+\alpha^{\prime} j 2(r)
\end{aligned}
$$

and $a 3(r), a 6(r), j 2(r), g 2(r), h 2(r)$ and $f 2(r)$ depends on higher derivative terms in the action and hence are computed purely from the background solution with $\alpha^{\prime} \rightarrow 0$. Among these coefficients $a 3$ is special, as, it couples to $\phi^{\prime \prime 2}$. All four derivatives act on the graviton fluctuation and thus $a 3$ only depends on metric functions in (2.2) and there r-derivatives. It is easy to convince ourselves that $a 3 \propto r\left(r^{2}-1\right)^{2} f\left(r, \alpha^{\prime}\right)$, where $f\left(r, \alpha^{\prime}\right)$ is a function that depend on the higher derivative terms and finite (constant or 0 ) at the boundary $r \rightarrow 0$. Now let us write the effective Lagrangian as follows,

$$
\begin{aligned}
S_{\mathrm{eff}}= & \frac{1+\alpha^{\prime} \Gamma}{16 \pi G_{5}} \int \frac{d^{4} k}{(2 \pi)^{4}} d r\left[\frac{4 r\left(r^{2}-1\right)^{2} \phi^{\prime}(r)^{2}-\omega^{2} \phi(r)^{2}}{4 r^{2}\left(r^{2}-1\right)}\right. \\
& \left.+\alpha^{\prime}\left(b 2(r) \phi(r)^{2}+b 1(r) \phi^{\prime}(r)^{2}\right)\right] .
\end{aligned}
$$

\footnotetext{
${ }^{22}$ In all the expressions we have omitted $k$ dependence of $\phi$ and other functions $a i^{\prime} s$ and $f 2, h 2, g 2, j 2$ and $b 1, b 2$ defined in next page.
} 
Demanding that the equation of motion (up to order $\alpha^{\prime}$ ) of $\phi$ derived from the original action and the above action are same we get,

$$
\begin{aligned}
b 1(r)= & \frac{1}{2 r\left(r^{2}-1\right)^{2}}\left[\left(-4 r^{3}-12 r+\omega^{2}\right) a 3(r)\right. \\
& +\left(r^{2}-1\right)\left(2 \kappa r^{4}-a 6^{\prime}(r) r^{3}-4 \kappa r^{2}+2 a 3^{\prime}(r) r^{2}\right. \\
& +2\left(r^{2}-1\right) h 2(r) r-2\left(r^{2}-1\right) j 2(r) r+a 6^{\prime}(r) r \\
& \left.\left.+2 \kappa+2 a 3^{\prime}(r)\right)\right] \\
b 2(r)= & -\frac{1}{16 r^{2}\left(r^{2}-1\right)^{4}}\left[\left(\omega^{4}+144 r^{3} \omega^{2}\right) a 3(r, k)\right. \\
& +4\left(r^{2}-1\right)\left(-4 r^{2} f 2(r)\left(r^{2}-1\right)^{3}\right. \\
& +\left(\left(\omega^{2} \kappa-2 r^{2}\left(r^{2}-1\right) j 2^{\prime \prime}(r)\right)\left(r^{2}-1\right)\right. \\
& \left.+2 r^{2} g 2^{\prime}(r)\left(r^{2}-1\right)^{2}+r \omega^{2} a 3^{\prime \prime}(r)\right)\left(r^{2}-1\right) \\
& \left.\left.+\left(1-11 r^{2}\right) \omega^{2} a 3^{\prime}(r)\right)\right] .
\end{aligned}
$$

The boundary terms coming from the original action (after adding Gibbons-Hawking boundary terms) are given by, ${ }^{23}$

$$
\begin{aligned}
S^{\mathcal{B}}= & \frac{1}{16 \pi G_{5}} \int \frac{d^{4} k}{(2 \pi)^{4}}\left[-\frac{\phi(r)^{2}}{r^{2}}+\phi(r)^{2}+r \phi^{\prime}(r) \phi(r)\right. \\
& -\frac{\phi^{\prime}(r) \phi(r)}{r}+\alpha^{\prime}\left(\frac{1}{2} g 2(r) \phi(r)^{2}-\frac{1}{2} j 2^{\prime}(r) \phi(r)^{2}\right. \\
& +\left(h 2(r)-j 2(r)-\frac{a 6^{\prime}(r)}{2}\right) \phi^{\prime}(r) \phi(r) \\
& +\frac{a 3^{\prime}(r)\left(\phi(r) \omega^{2}+4\left(r^{4}-1\right) \phi^{\prime}(r)\right) \phi(r)}{4 r\left(r^{2}-1\right)^{2}} \\
& -\frac{a 3(r)\left(6 r \phi(r) \phi^{\prime}(r) \omega^{2}\right)}{4 r\left(r^{2}-1\right)^{3}} \\
& -\frac{a 3(r)\left(\left(r^{2}-1\right)\left(8 r^{3}+24 r-\omega^{2}\right) \phi^{\prime}(r)\right) \phi(r)}{4 r\left(r^{2}-1\right)^{3}} \\
& -\frac{a 3(r) \phi^{\prime}(r)\left(\phi(r) \omega^{2}+4\left(r^{4}-1\right) \phi^{\prime}(r)\right)}{4 r\left(r^{2}-1\right)^{2}} \\
& \left.\left.-a 3(r) \phi^{\prime}(r)\left(-\frac{\phi(r) \omega^{2}}{2 r\left(r^{2}-1\right)^{2}}-\frac{\left(r^{4}-1\right) \phi^{\prime}(r)}{r\left(r^{2}-1\right)^{2}}\right)\right)\right]
\end{aligned}
$$

\footnotetext{
${ }^{23}$ There was a sign error in [29].
} 
And the boundary terms coming from the effective action are given by,

$$
\begin{aligned}
S_{\text {seff }}^{\mathcal{B}}= & \frac{1}{16 \pi G_{5}} \int \frac{d^{4} k}{(2 \pi)^{4}}\left[\left(r-\frac{1}{r}\right) \phi(r) \phi^{\prime}(r)\right. \\
& +\frac{\alpha^{\prime}}{2 r\left(r^{2}-1\right)^{2}}\left(\phi ( r ) \left(2 \Gamma\left(r^{2}-1\right)^{3}+\left(-a 6^{\prime}(r) r^{3}\right.\right.\right. \\
& +2 a 3^{\prime}(r) r^{2}+2\left(r^{2}-1\right) h 2(r) r-2\left(r^{2}-1\right) j 2(r) r \\
& \left.+a 6^{\prime}(r) r+2 a 3^{\prime}(r)\right)\left(r^{2}-1\right) \\
& \left.\left.\left.+\left(-4 r^{3}-12 r+\omega^{2}\right) a 3(r)\right) \phi^{\prime}(r)\right)\right] .
\end{aligned}
$$

Now, it is interesting to compute the difference between these two boundary terms and the result is, ${ }^{24}$

$$
\begin{aligned}
S^{\mathcal{B}}-S_{\text {eff }}^{\mathcal{B}}=\frac{1}{16 \pi G_{5}} \int & \frac{d^{4} k}{(2 \pi)^{4}}\left[-\frac{\phi(r)^{2}}{r^{2}}+\phi(r)^{2}\right. \\
& +\alpha^{\prime}\left(\frac{1}{2} g 2(r) \phi(r)^{2}-\frac{1}{2} j 2^{\prime}(r) \phi(r)^{2}\right. \\
& \left.\left.+\frac{a 3^{\prime}(r) \omega^{2} \phi(r)^{2}}{4 r\left(r^{2}-1\right)}-\frac{a 3(r)\left(6 r \omega^{2}\right) \phi(r)^{2}}{4 r\left(r^{2}-1\right)^{3}}\right)\right]
\end{aligned}
$$

The term proportional to $a 3$ in the parenthesis of (B.8) vanishes at the boundary whereas the term proportional to $a 3^{\prime}$ gives a pure UV divergent piece and a vanishing piece, due to the property of $a 3$ mentioned above. This is true irrespective of the choice of the higher derivative terms. Thus, we see that the two boundary terms differ only by terms which are either purely divergent or of the form $g(r) \phi^{2}$, where $g(r)$ is any function of $r$. The divergent terms would get canceled once appropriate boundary terms are added (which has been discussed in sections 2 and 3). The terms proportional to $\phi^{2}$ can only contribute to pressure of the boundary theory $\left(g 2(r)\right.$ and $j 2^{\prime}(r)$ can have terms proportional to momentum, but those terms are either divergent or vanish at the boundary $\left.{ }^{25}\right)$. and are

\footnotetext{
${ }^{24}$ It has been shown in [29] that $\Gamma=0$.

${ }^{25}$ They have following structure,

$$
g 2 \sim(\text { momenta })^{2}\left(\frac{A}{r}+B r\right)\left(C+r^{m}\right)
$$

and

$$
j 2 \sim(\text { momenta })^{2}\left(D+r^{m}\right),
$$

where, $A, B, C, D$ are constants depending on the higher derivative term. The constant $m \geq 2$. The above mentioned structure of the two functions can be derived from the structure of the Riemann tensor. Let us first concentrate on $g 2$. In a $R^{n}$ higher derivative gravity, there are total $2 \mathrm{n}$ number o derivatives acting on the metric. From (B.1), we see that $g 2$ appears in $a 4(r)$ which is a coefficient of $\phi(r) \phi^{\prime}(r)$. Moreover, we are looking for the piece of $g 2$ which is proportional to the squre of the momenta $\left(\omega^{2}\right.$ or $\left.q^{2}\right)$. This requires total three derivatives (one $\mathrm{r}$ and two $\mathrm{t} / \mathrm{z}$ ) acting on graviton fluctuation $\phi(r, t, z)$. All other $(2 n-3)$ derivatives will act on the background metric and as the background metric is only function of $r$, the derivatives will be only with respect to $r$. The structure of the Riemann tensor $R_{b c d}^{a}$ is as follows:
}

$$
\text { Riemann } \sim \Gamma \Gamma-\partial \Gamma \sim g^{-1} g^{-1} \partial g \partial g+g^{-1} \partial^{2} g,
$$


not important for the computation of transport coefficients of the boundary plasma. Thus we see that, it is obvious that the transport coefficients coming from the original action and the boundary action are same.

Here we have considered only $R^{(n)}$ gravity theory. A more rigorous proof is required for theories involving covariant derivatives of curvature tensors and scalars.

\section{Functions appeared in String theory corrected action}

$$
\begin{aligned}
\mathcal{A}_{0}^{W^{4}}(r, k)= & -\frac{r_{0}^{2}\left(q^{2}\left(r^{2}-1\right)+\omega^{2}\right)}{4 r^{2}\left(r^{2}-1\right)} \\
& -\frac{1}{4\left(r^{2}-1\right)}\left(\gamma \left(4 q^{2} r^{2}\left(\left(245 r^{4}-407 r^{2}+162\right) r_{0}^{2}+10 r \omega^{2}\right)\right.\right. \\
& \left.\left.+3\left(221 r^{4}-191 r^{2}+25\right) \omega^{2} r_{0}^{2}\right)\right)
\end{aligned}
$$

and

$$
\mathcal{A}_{1}^{W^{4}}(r, k)=\frac{\left(r^{2}-1\right) r_{0}^{4}}{r}+3 r\left(r^{2}-1\right) \gamma r_{0}^{2}\left(\left(43 r^{4}+47 r^{2}-25\right) r_{0}^{2}+16 q^{2} r^{3}\right)
$$

\section{Functions appeared in four derivative action}

$$
\begin{aligned}
\mathcal{A}_{0}^{\mathrm{GB}}(r, k)= & -\frac{q^{2}\left(r^{2}-1\right)+\omega^{2}}{4 r^{2}\left(r^{2}-1\right)} \\
& +\frac{\alpha^{\prime}}{12 r^{2}\left(r^{2}-1\right)}\left(q ^ { 2 } \left(2 \beta_{3}\left(13 r^{2}-3 r \omega^{2}-13\right)\right.\right. \\
& \left.+130\left(r^{2}-1\right) \beta_{1}+\left(-36 r^{4}+25 r^{2}+11\right) \beta_{2}\right) \\
& \left.+\omega^{2}\left(\left(6 r^{2}-11\right) \beta_{2}+130 \beta_{1}+26 \beta_{3}\right)\right)
\end{aligned}
$$

and

$$
\mathcal{A}_{1}^{\mathrm{GB}}(r, k)=r-\frac{1}{r}-\frac{\left(r^{2}-1\right)\left(\left(18 r^{2}-13\right) \beta_{2}+110 \beta_{1}+22 \beta_{3}\right) \alpha^{\prime}}{3 r} .
$$

where, $g^{-1}$ means inverse metric. We have not put explicit indices here as that would not be important. Ricci tensor also has the same structure and Ricci scalar terms are not required (they never contribute to $\left.\phi(r) \phi^{\prime}(r)\right)$. Then it is easy to see that for any $R^{n}$ higher derivative terms, only two Reimann tensors will contribute to $\phi(r) \phi^{\prime}(r)$ and others $n-2$ have to be computed on the background metric. Moreover form (B.11), only the product of the first piece and the second piece coming from two different Riemann tensor will give $\phi(r) \phi^{\prime}(r)$. From this generic structure we get the result (B.9). The first parenthesis in (B.9) comes from the two Riemann tensors while the second one gives the contribution from all others.

Similarly, we see that $j 2$ appears in $a 5(r)$ which is a coefficient of $\phi(r) \phi^{\prime \prime}(r)$. Moreover, we are looking for the piece of $j 2$ which is proportional to $\omega^{2}\left(o r q^{2}\right)$. This requires total four derivatives (two $\mathrm{r}$ and two $\mathrm{t} / \mathrm{z}$ ) acting on graviton fluctuation $\phi(r, t, z)$. All other $(2 n-4)$ derivatives will act on the background metric and as mentioned earlier, the derivatives will be only with respect to $r$. From (B.11), only the product of the second piece coming from two different Riemann tensor will give $\phi(r) \phi^{\prime \prime}(r)$. From this generic structure we get the result (B.10). 


\section{E Functions appeared in Higher-derivative Charged black-hole action}

$$
\begin{aligned}
\mathcal{A}_{1}^{C B}= & \frac{r^{5}\left(\omega^{2}-q^{2}\right)+q^{2} r_{0}^{4} r}{2\left(r^{4}-r_{0}^{4}\right)} \\
& +\frac{c_{1} \alpha^{\prime}\left(11 r^{8}\left(\omega^{2}-q^{2}\right)-r_{0}^{4} r^{4}\left(25 q^{2}+6 \omega^{2}\right)+36 q^{2} r_{0}^{8}\right)}{6 r^{3}\left(r^{4}-r_{0}^{4}\right)} \\
& +\frac{Q^{2}}{2 r_{0}^{2}\left(r^{2}-r_{0}^{2}\right)\left(r^{2}+r_{0}^{2}\right)^{2}}\left[r^{3} \omega^{2}+\frac{\alpha^{\prime}}{3 r^{5}}\left(c _ { 1 } \left[28 q^{2} r_{0}^{6} r^{2}\right.\right.\right. \\
& -r^{8}\left(36 q^{2}+127 \omega^{2}\right)-4 r_{0}^{2} r^{6}\left(7 q^{2}-3 \omega^{2}\right)-8 q^{2} r_{0}^{8} \\
& \left.+4 r_{0}^{4} r^{4}\left(11 q^{2}+3 \omega^{2}\right)\right]-24 c_{2}\left[r_{0}^{2} r^{6}\left(2 q^{2}-3 \omega^{2}\right)-2 q^{2} r_{0}^{6} r^{2}\right. \\
& \left.\left.\left.+r_{0}^{4} r^{4}\left(2 q^{2}-3 \omega^{2}\right)-2 q^{2} r_{0}^{8}+4 r^{8} \omega^{2}\right]\right)\right]+\mathcal{O}\left(Q^{4}\right)
\end{aligned}
$$

and

$$
\begin{aligned}
\mathcal{A}_{1}^{C B}= & \frac{1}{2}\left(r r_{0}^{4}-r^{5}\right)-\frac{c_{1} \alpha^{\prime}\left(13 r^{4}-18 r_{0}^{4}\right)\left(r^{4}-r_{0}^{4}\right)}{6 r^{3}} \\
& +\frac{Q^{2}\left(r^{2}-r_{0}^{2}\right)}{2 r r_{0}^{2}}\left[1-\frac{\alpha^{\prime}}{3 r^{4}}\left(24 c_{2}\left(4 r^{4}-3 r_{0}^{2} r^{2}-3 r_{0}^{4}\right)\right.\right. \\
& \left.\left.+c_{1}\left(101 r^{4}-156 r_{0}^{2} r^{2}-120 r_{0}^{4}\right)\right)\right]+\mathcal{O}\left(Q^{4}\right) .
\end{aligned}
$$

Open Access. This article is distributed under the terms of the Creative Commons Attribution Noncommercial License which permits any noncommercial use, distribution, and reproduction in any medium, provided the original author(s) and source are credited.

\section{References}

[1] G. Policastro, D.T. Son and A.O. Starinets, The shear viscosity of strongly coupled $N=4$ supersymmetric Yang-Mills plasma, Phys. Rev. Lett. 87 (2001) 081601 [hep-th/0104066] [SPIRES].

[2] D.T. Son and A.O. Starinets, Viscosity, black holes and quantum field theory, Ann. Rev. Nucl. Part. Sci. 57 (2007) 95 [arXiv:0704.0240] [SPIRES].

[3] P. Kovtun, D.T. Son and A.O. Starinets, Viscosity in strongly interacting quantum field theories from black hole physics, Phys. Rev. Lett. 94 (2005) 111601 [hep-th/0405231] [SPIRES].

[4] P.K. Kovtun and A.O. Starinets, Quasinormal modes and holography, Phys. Rev. D 72 (2005) 086009 [hep-th/0506184] [SPIRES].

[5] D.T. Son and A.O. Starinets, Minkowski-space correlators in AdS/CFT correspondence: Recipe and applications, JHEP 09 (2002) 042 [hep-th/0205051] [SPIRES].

[6] C.P. Herzog and D.T. Son, Schwinger-Keldysh propagators from AdS/CFT correspondence, JHEP 03 (2003) 046 [hep-th/0212072] [SPIRES]. 
[7] R.A. Janik and R.B. Peschanski, Asymptotic perfect fluid dynamics as a consequence of AdS/CFT, Phys. Rev. D 73 (2006) 045013 [hep-th/0512162] [SPIRES].

[8] R.A. Janik and R.B. Peschanski, Gauge/gravity duality and thermalization of a boostinvariant perfect fluid, Phys. Rev. D 74 (2006) 046007 [hep-th/0606149] [SPIRES].

[9] S. Bhattacharyya, V.E. Hubeny, S. Minwalla and M. Rangamani, Nonlinear fluid dynamics from gravity, JHEP 02 (2008) 045 [arXiv:0712.2456] [SPIRES].

[10] R. Baier, P. Romatschke, D.T. Son, A.O. Starinets and M.A. Stephanov, Relativistic viscous hydrodynamics, conformal invariance and holography, JHEP 04 (2008) 100 [arXiv:0712.2451] [SPIRES].

[11] M. Natsuume and T. Okamura, Causal hydrodynamics of gauge theory plasmas from AdS/CFT duality, Phys. Rev. D 77 (2008) 066014 [Erratum ibid. D 78 (2008) 089902] [arXiv: 0712.2916] [SPIRES].

[12] M. Haack and A. Yarom, Nonlinear viscous hydrodynamics in various dimensions using AdS/CFT, JHEP 10 (2008) 063 [arXiv:0806.4602] [SPIRES].

[13] S. Bhattacharyya, R. Loganayagam, I. Mandal, S. Minwalla and A. Sharma, Conformal nonlinear fluid dynamics from gravity in arbitrary dimensions, JHEP 12 (2008) 116 [arXiv: 0809.4272] [SPIRES].

[14] M. Van Raamsdonk, Black hole dynamics from atmospheric science, JHEP 05 (2008) 106 [arXiv: 0802.3224] [SPIRES].

[15] R. Loganayagam, Entropy current in conformal hydrodynamics, JHEP 05 (2008) 087 [arXiv:0801.3701] [SPIRES].

[16] M.P. Heller, P. Surowka, R. Loganayagam, M. Spalinski and S.E. Vazquez, On a consistent AdS/CFT description of boost-invariant plasma, arXiv:0805.3774 [SPIRES].

[17] I.P. Neupane and N. Dadhich, Higher curvature gravity: entropy bound and causality violation, arXiv:0808.1919 [SPIRES].

[18] J.J. Friess, S.S. Gubser, G. Michalogiorgakis and S.S. Pufu, Expanding plasmas and quasinormal modes of anti-de Sitter black holes, JHEP 04 (2007) 080 [hep-th/0611005] [SPIRES].

[19] C.P. Herzog, The hydrodynamics of M-theory, JHEP 12 (2002) 026 [hep-th/0210126] [SPIRES].

[20] G. Policastro, D.T. Son and A.O. Starinets, From AdS/CFT correspondence to hydrodynamics. II: Sound waves, JHEP 12 (2002) 054 [hep-th/0210220] [SPIRES].

[21] R.K. Gupta and A. Mukhopadhyay, On the universal hydrodynamics of strongly coupled CFTs with gravity duals, JHEP 03 (2009) 067 [arXiv:0810.4851] [SPIRES].

[22] S.S. Pal, Weak gravity conjecture, central charges and $\eta / s$, arXiv:1003.0745 [SPIRES].

[23] S.S. Pal, $\eta /$ s at finite coupling, Phys. Rev. D 81 (2010) 045005 [arXiv:0910.0101] [SPIRES].

[24] M.F. Paulos, Transport coefficients, membrane couplings and universality at extremality, JHEP 02 (2010) 067 [arXiv:0910.4602] [SPIRES] [SPIRES].

[25] C.P. Herzog, The sound of M-theory, Phys. Rev. D 68 (2003) 024013 [hep-th/0302086] [SPIRES].

[26] R.H. Price and K.S. Thorne, Membrane viewpoint on black holes: properties and evolution of the stretched horizon, Phys. Rev. D 33 (1986) 915 [SPIRES]. 
[27] N. Iqbal and H. Liu, Universality of the hydrodynamic limit in AdS/CFT and the membrane paradigm, Phys. Rev. D 79 (2009) 025023 [arXiv:0809.3808] [SPIRES].

[28] R.G. Cai, Z.Y. Nie and Y.W. Sun, Shear viscosity from effective couplings of gravitons, Phys. Rev. D 78 (2008) 126007 [arXiv:0811.1665] [SPIRES].

[29] N. Banerjee and S. Dutta, Higher derivative corrections to shear viscosity from graviton's effective coupling, JHEP 03 (2009) 116 [arXiv:0901.3848] [SPIRES].

[30] N. Banerjee and S. Dutta, Shear viscosity to entropy density ratio in six derivative gravity, JHEP 07 (2009) 024 [arXiv: 0903.3925] [SPIRES].

[31] N. Banerjee and S. Dutta, Near-horizon analysis of $\eta / s$, arXiv:0911.0557 [SPIRES].

[32] A. Buchel and M. Paulos, Relaxation time of a CFT plasma at finite coupling, Nucl. Phys. B 805 (2008) 59 [arXiv: 0806.0788] [SPIRES].

[33] R.C. Myers, M.F. Paulos and A. Sinha, Holographic hydrodynamics with a chemical potential, JHEP 06 (2009) 006 [arXiv: 0903.2834] [SPIRES].

[34] K. Skenderis, Lecture notes on holographic renormalization, Class. Quant. Grav. 19 (2002) 5849 [hep-th/0209067] [SPIRES].

[35] S. de Haro, S.N. Solodukhin and K. Skenderis, Holographic reconstruction of spacetime and renormalization in the AdS/CFT correspondence, Commun. Math. Phys. 217 (2001) 595 [hep-th/0002230] [SPIRES].

[36] S.S. Gubser, I.R. Klebanov and A.A. Tseytlin, Coupling constant dependence in the thermodynamics of $N=4$ supersymmetric Yang-Mills theory, Nucl. Phys. B 534 (1998) 202 [hep-th/9805156] [SPIRES].

[37] S. Dutta and R. Gopakumar, On Euclidean and Noetherian entropies in AdS space, Phys. Rev. D 74 (2006) 044007 [hep-th/0604070] [SPIRES].

[38] E.A. Bergshoeff and M. de Roo, The quartic effective action of the heterotic string and supersymmetry, Nucl. Phys. B 328 (1989) 439 [SPIRES].

[39] W.A. Chemissany, M. de Roo and S. Panda, $\alpha^{\prime}$-corrections to heterotic superstring effective action revisited, JHEP 08 (2007) 037 [arXiv:0706.3636] [SPIRES].

[40] Y. Kats and P. Petrov, Effect of curvature squared corrections in AdS on the viscosity of the dual gauge theory, JHEP 01 (2009) 044 [arXiv:0712.0743] [SPIRES].

[41] M. Blau, K.S. Narain and E. Gava, On subleading contributions to the AdS/CFT trace anomaly, JHEP 09 (1999) 018 [hep-th/9904179] [SPIRES].

[42] S. Dutta, Higher derivative corrections to locally black brane metrics, JHEP 05 (2008) 082 [arXiv: 0804.2453] [SPIRES].

[43] M. Brigante, H. Liu, R.C. Myers, S. Shenker and S. Yaida, Viscosity bound violation in higher derivative gravity, Phys. Rev. D 77 (2008) 126006 [arXiv:0712.0805] [SPIRES].

[44] A. Buchel et al., Holographic GB gravity in arbitrary dimensions, JHEP 03 (2010) 111 [arXiv: 0911.4257] [SPIRES].

[45] A. Buchel and R.C. Myers, Causality of holographic hydrodynamics, JHEP 08 (2009) 016 [arXiv: 0906.2922] [SPIRES].

[46] M. Cvetič and S.S. Gubser, Phases of R-charged black holes, spinning branes and strongly coupled gauge theories, JHEP 04 (1999) 024 [hep-th/9902195] [SPIRES]. 
[47] A. Chamblin, R. Emparan, C.V. Johnson and R.C. Myers, Holography, thermodynamics and fluctuations of charged AdS black holes, Phys. Rev. D 60 (1999) 104026 [hep-th/9904197] [SPIRES].

[48] R.-G. Cai and A. Wang, Thermodynamics and stability of hyperbolic charged black holes, Phys. Rev. D 70 (2004) 064013 [hep-th/0406057] [SPIRES].

[49] S.S. Gubser, Thermodynamics of spinning D3-branes, Nucl. Phys. B 551 (1999) 667 [hep-th/9810225] [SPIRES].

[50] N. Banerjee and S. Dutta, Phase transition of electrically charged Ricci-flat black holes, JHEP 07 (2007) 047 [arXiv: 0705. 2682] [SPIRES].

[51] N. Banerjee et al., Hydrodynamics from charged black branes, arXiv:0809.2596 [SPIRES].

[52] J. Erdmenger, M. Haack, M. Kaminski and A. Yarom, Fluid dynamics of R-charged black holes, JHEP 01 (2009) 055 [arXiv:0809.2488] [SPIRES].

[53] D.T. Son and A.O. Starinets, Hydrodynamics of R-charged black holes, JHEP 03 (2006) 052 [hep-th/0601157] [SPIRES].

[54] K. Maeda, M. Natsuume and T. Okamura, Viscosity of gauge theory plasma with a chemical potential from AdS/CFT, Phys. Rev. D 73 (2006) 066013 [hep-th/0602010] [SPIRES].

[55] S. Cremonini, K. Hanaki, J.T. Liu and P. Szepietowski, Higher derivative effects on eta/s at finite chemical potential, Phys. Rev. D 80 (2009) 025002 [arXiv: 0903. 3244] [SPIRES].

[56] E. D'Hoker and P. Kraus, Charged magnetic brane solutions in AdS $S_{5}$ and the fate of the third law of thermodynamics, JHEP 03 (2010) 095 [arXiv:0911.4518] [SPIRES]. 